\title{
A novel network approach to study communication activities of air traffic controllers
}

\author{
Yanjun Wang ${ }^{\mathrm{a}, \mathrm{b}}$, Jian Bu ${ }^{\mathrm{a}, \mathrm{b}}$, Ke Han ${ }^{\mathrm{c}, *}$, Rui Sun ${ }^{\mathrm{a}, \mathrm{b}}$, Minghua Hu ${ }^{\mathrm{a}, \mathrm{b}}$, Chenping $\mathrm{Zhu}^{\mathrm{d}}$ \\ ${ }^{a}$ College of Civil Aviation, Nanjing University of Aeronautics and Astronautics, Nanjing 210016, China \\ ${ }^{b}$ National Key Laboratory of Air Traffic Flow Management, Nanjing 210016, China \\ ${ }^{c}$ Department of Civil and Environmental Engineering, Imperial College London, SW7 2BU, UK \\ ${ }^{d}$ Department of Physics, Nanjing University of Aeronautics and Astronautics, Nanjing 210016, China
}

\begin{abstract}
Air traffic controllers play critical roles in the safety, efficiency, and capacity of air traffic management. However, there is inadequate knowledge of the dynamics of the controllers' activities, especially from a quantitative perspective. This paper presents a novel network approach to uncover hidden patterns of the controller's behavior based on the voice communication data. We convert the time series of the controller's communication activities, which contain flights' information, into a time-varying network and a static network, referred to as temporal network and timeaggregated network, respectively. These networks reflect the interaction between the controller and the flights on a sector level, and allow us to leverage network techniques to yield new and insightful findings regarding regular patterns and unique characteristics of the controller's communication activities. The proposed methodology is applied to three real-world datasets, and the resulting networks are closely examined and compared in terms of degree distribution, community structure, and motifs. This network approach introduces a "spatial" element to the conventional analysis of the controllers' communication events, by identifying connectivity and proximity among flights. It constitutes a major step towards the quantitative description of the controller-flight dynamics, which is not widely seen in the literature.
\end{abstract}

Keywords:

Air traffic management, air traffic controller, communication events, network science

\section{Introduction}

The past decade has seen a significant improvement in the air traffic management (ATM) system in terms of safety, capacity, and efficiency. Great efforts have been made to enhance the performance of the ATM system, including the introduction of new operational concepts and protocols, deployment of advanced automation systems, and strategic research and development activities. Despite the ongoing exercise of new operational concepts and deployment of technologies in both Single European Sky ATM Research (SESAR) in Europe, and Next Generation Air Transportation System (NextGen) in the US, air traffic controllers are, and continue to be, playing critical roles in the ATM system. The in-depth understanding of the controllers' activities remains critical to ensure the safety and efficiency of the ATM system.

In many problems arising from human-driven complex systems, it is necessary to evaluate the operator's activities. Among various internal and external activities, mental workload has been the central focus of investigation in ATM-related research. Subjective measures, performance metrics, as well as psychophysiological indices, are often used to assess a particular type of cognitive load, which contributes to the workload (Galy et al., 2012). Along this line of research, earlier work was based on queuing theory and examination of controller's routine work (Schmidt,

\footnotetext{
${ }^{*}$ Corresponding author

Email addresses: ywang@nuaa.edu.cn (Yanjun Wang), bj@nuaa.edu.cn (Jian Bu), k.han@imperial.ac.uk (Ke Han), rui. sun@nuaa.edu.cn (Rui Sun), minghuahu@nuaa. edu. cn (Minghua Hu), chenpingzhu@aliyun. com (Chenping Zhu)
} 
1978), while more recent approaches employ the controller's subjective rating (Manning et al., 2002). In the attempt to prevent high workload, studies have focused on relevant factors that influence the workload. For example, many researchers have demonstrated that complexity factors can reduce sector capacity by increasing the controllers' workload (Loft et al., 2007; Laudeman et al., 1998; Sridhar et al., 1998; Averty et al., 2004; Hilburn, 2004). As the ATM system is undergoing a transformative development, there are ongoing attempts to analyze the controllers' activities in a futuristic operational environment. Human-in-the-loop studies have been conducted either to develop new cognitive metrics, or to evaluate decision support tools that incorporate future operational concepts (Li and Hansman, 2009; Kupfer et al., 2011; Guo et al., 2014). Issues that could rise while implementing key operational changes, such as trajectory-based operation and data communication, have been progressively identified (Lacher et al., 2011). Although there are extensive studies on controllers' workload and other topics related to human factors, the quantitative characterization and assessment of controllers' activities remain an open question and rarely studied. The limited knowledge about the underlying dynamics of air traffic controllers and the lack of proper mathematical tools have prevented us from an in-depth investigation of the controllers' activities. Conventional methods tend to target some specific problems, e.g. the analysis of controllers' workload in a certain sector. With a few exceptions (Clarke et al., 2011; Histon and Hansman Jr, 2008), much less has been done regarding the understanding of the dynamics of air traffic controllers' activities.

In the past few years there has been a surge of interest in both empirical studies of human daily activities and development of models to explain observed phenomena (Oliveira and Barabási, 2005; Barabási, 2005; Malmgren et al., 2009). Burgeoning empirical evidence regarding human dynamics recently uncovered similar patterns among human being, which suggests that there exist universal mechanisms that govern human activities. Investigation of large empirical datasets of human activities, including mail correspondence (Oliveira and Barabási, 2005), email communication (Malmgren et al., 2008), text messaging, and online film rating (Zhou et al., 2008), indicates that the temporal patterns of human actions exhibit bursts of frequent actions separated by long periods of inactivity. Quantitative assessments on the circulation of bank notes (Brockmann et al., 2006) and mobile phone datasets (Gonzalez et al., 2008; Song et al., 2010) demonstrate that the human trajectories show a high degree of temporal and spatial regularities. Researchers have developed various models to reveal the fundamental rules that human follow when executing tasks.

We note that, in contrast to the activities examined in the aforementioned studies on human dynamics, people in safety-critical or mission-critical systems (e.g. air traffic management) are typically under pressure and stress. In ATM, any mistake made by the controller could cause huge economics cost, or even loss of lives. Therefore, the understanding of air traffic controllers' behavioral dynamics is vital to the safety and efficiency of air traffic management systems.

Although controllers' activities are influenced by contextual factors such as airspace structure and air traffic flow distribution, cognitive analyses have uncovered several common strategies employed by controllers while handling traffic (Histon and Hansman Jr, 2008). However, whether there is a similar mechanism behind the controllers' activities, which holds for normal daily activities, remains unanswered, partially due to the lack of empirical data and proper analysis tools. Instead, most studies on relevant topics mainly rely on the assumption of a priori random distributions that the controllers' behavioral parameters follow, such as Poisson distribution, lognormal distribution, and Weibull distribution (Alam et al., 2013; Yang and Hu, 2010; Gravio et al., 2015; Prasad and Gaikwad, 2015).

Researchers from the fields of cognitive science and psychology have undertaken significant work in the attempt to analyze and understand, in a qualitative way, controllers' behavior, workload, and capacity. For example, Histon and Hansman Jr (2008) report four strategies that air traffic controllers adapt in order to reduce the cognitive complexity: (1) standard flow; (2) critical points; (3) grouping; and (4) responsibility. However, no quantifiable outcomes regarding the controllers' behavioral dynamics are provided.

This paper aims to uncover the hidden patterns of controllers behavior from their voice communication data, in a novel and quantitative way by leveraging network techniques. In particular, we propose a network approach to uncover the underlying mechanisms of controllers' activities and quantify the dynamics of the controller-flight interaction. Networks are widely used to represent the patterns of connections among various components of complex systems. Recently, there is hightened interest in the study of temporal networks, which fuse the temporal dimension into the classical network analyses (Chechik et al., 2008; Ahmed and Xing, 2009; Holme and Saramäki, 2011). For example, temporal networks have been used to study the propagation of information in social networks (Liben-Nowell and Kleinberg, 2008; Rocha et al., 2010), and extract information from web posts to analyze prostitution activities (Rocha et al., 2010). Techniques are employed to convert time series to graphs, followed by network analyses, in 
order to unmask the hidden dynamics; see Zhang and Small (2006); Xu et al. (2008); Vassilis (2009); Haraguchi et al. (2009) and Gautreau et al. (2009). According to DONNER et al. (2010), methods that convert time series to network structures can be classified into three categories :

(i) Mutual proximity of different segments of a time series. For example, in the recurrence plot approach, nodes are defined from the phase space trajectory and a link between two cycles when two nodes are rather similar (Marwan et al., 2009);

(ii) Convexity of successive observations (i.e. visibility graphs). Algorithm for converting time series into a graph can be found in (Lacasa et al., 2008);

(iii) Transition probabilities between discrete states (transition networks) (Campanharo et al., 2011).

The time series of controllers' voice communication (or more recently, data communication) events contain rich information about the underlying mechanisms of their activities, since voice/text communication is the only channel for the flow of information between the controller and the pilots. Inspired by previous analyses of the temporal characteristics of controller's activities (Wang et al., 2013, 2016), in this paper we investigate their "spatial behavior" using a network approach, by introducing notions of connectivity and proximity. In this work, we first present a method that converts time series data into a time-dependent network, referred to as temporal network, which is then further aggregated over time to obtain a static network, called time-aggregated network.

In the time-aggregated network, we focus on network metrics such as degree distribution and community structure ${ }^{1}$, which provides interesting and insightful findings regarding the controller's communication pattern. On the other hand, while there are abundant measures for characterizing the topological structure of static networks, measures proposed for the temporal networks are scarce. Most of them are based on the concept of time-respecting paths that define the accessibility of nodes from other nodes within some observation window; examples include reachability ratio, connectivity, distances, and latencies (Holme and Saramäki, 2011). Given the unique characteristics of air traffic control, we propose to analyze the temporal network from the perspectives of time-dependent degree distribution and network motifs, which enable us to capture the temporal dynamics on a sector level.

Specific contribution and findings made by this paper are as follows.

- We propose a new method to convert time series of controller's voice communication events into two types of networks: a time-varying network and a time-aggregated network. This allows conventional notions and techniques from network science to be applied to yield new and insightful findings regarding controller's activities.

- The proposed network framework has led to the following main findings.

- In the time-aggregated network, the structure of the communities is highly correlated to the dynamic traffic flow in the sector, which confirms the controllers' communication activities being coupled with the traffic in real time.

- The aggregated network without any filtering of noises exhibits Poisson-like degree distribution, which suggests local randomness in the selection of flights when it comes to the controller's communication activity. After we filter the noises (i.e. minor communication event such as confirmation or acknowledgement of transmission), the degree distribution becomes heavily left-skewed, which means a few flights get constant attention from the controller while the remaining do not (and are randomly selected).

- In the temporal network, most flights have a degree of 2. It is also found that chains and loops are the most common motifs in the controllers' communication, and part of them evolve into stars as time goes on. To the best of our knowledge, this is the first quantification of the grouping behavior reported in Histon and Hansman Jr (2008).

- In terms of time-dependent degree distribution of the temporal networks, we found similar shapes of the distributions across all sectors and repetitions of exercise. This reveals a regular communication pattern that is independent of the airspace structure.

\footnotetext{
${ }^{1}$ Communities are defined to be dense sub-networks within a larger network, which collectively reflect certain structure of the network
} 
- Regarding network motif ${ }^{2}$, it is found that the most commonly seen communication patterns are chains and loops. When the observational period increases, most chains ultimately form loops; and only about $20 \%$ of the motifs are stars.

- This paper is among the first to identify regular patterns in air traffic controllers' communication activities which, according to our literature review above, is quite scarce for mission-critical activities under pressure. The analytical framework put forward may be also applied to other task-driven activities.

The work described in this paper introduces a new "spatial" perspective to the analysis of controllers' temporal communication events, by identifying connectivity and proximity among flights. Moreover, by leveraging network dynamics, we are able to uncover regular communication patterns of controllers and the dynamics of their behavior, which constitutes a major step towards quantitative description of the controller-flight dynamics. Furthermore, the proposed framework involving network analyses could bring transformative changes to the way in which controllers' activities are currently assessed.

With regard to the future air traffic operation, in which automation might play an increasingly important role, the study described by this paper has a profound impact since the underlying communication patterns uncovered through the proposed network approach not only help to better understand human activities in mission-critical systems, but also provide support to the development of automation systems. This is because the controller's objective is to maintain a safe and orderly flow of air traffic by sending control instructions to pilots via communication systems, which amounts to an information diffusion process. With the increased knowledge of the controllers dynamics, we are able to support the development of automation systems with similar information diffusion behavior for various operational environments.

The rest of the paper is organized as follows. Section 2 presents our methods to convert a series of communication events into networks. In Section 3 we describe the empirical datasets used in this study. In Section 4 we conduct comprehensive analysis of the datasets mentioned in Section 3 using the proposed network approach, and provide unique findings and insights by analyzing the degree distribution, community, and motifs of the networks. Finally, Section 5 provides some concluding remarks.

\section{Converting Communication Events into Networks}

The table below summarizes the main notations employed in this paper.

\begin{tabular}{ll}
\hline$T_{\min }$ & beginning of the time horizon \\
$T_{\max }$ & end of the time horizon \\
$F_{i}$ & Flight $i$ \\
$\tau_{i}(t)$ & start time of the latest call event for $F_{i}$ relative to time $t$ \\
$l_{i}(t)$ & duration of the latest call for $F_{i}$ \\
$D(i, j ; t)$ & the temporal distance between $F_{i}$ and $F_{j}$ at time $t$ \\
$I_{1}, \ldots, I_{n}$ & partition of the entire horizon into $n$ equal subintervals \\
$\tau_{t w}$ & length of each of the $n$ subintervals \\
$\tau_{\min }$ & prescribed threshold to determine the connection between two flights \\
$N_{\min }$ & prescribed integer threshold to filter random connections between two flights \\
\hline
\end{tabular}

\subsection{Information transmission via controller's voice communication}

Within the framework of air traffic management, an airspace is partitioned into smaller parts called sectors. Each sector is assigned one or two air traffic controllers who are responsible for the safety and efficiency of air traffic flow. The controllers send instructions and clearances to the pilots regarding heading, speed, and altitude, in order to

\footnotetext{
${ }^{2}$ A network motif is the equivalence class of subgraphs or patterns that recur much more often than randomly. In this paper we consider three types of motifs: chain, loop, and star.
} 
maintain a safe and orderly flow of aircrafts. Voice communication is the most common way for controllers and pilots to exchange information. This paper focuses on the radar control, in which voice communication is the only chancel between the controllers and pilots. With such a premise, it can be assumed that the controller's voice communication activity encapsulates both cognitive and physical efforts to accomplish the mission of air traffic control.

The controller needs to press the push-to-talk (PTT) button in order to send radio transmissions to the aircraft. Normally the content of the transmission should contain information as to which aircraft the communication is addressing. One controller communication event is defined as the period when the controller presses the PTT button before releasing it. Figure 1 provides an example of the communication activities of a controller recorded in a 15-minute interval. This figure shows that the controller tends to talk to a group of flights, followed by a short period of silence, and his/her interaction with different flights seems random. In order to uncover hidden patterns and information from the time series data, we introduce the network approach below.

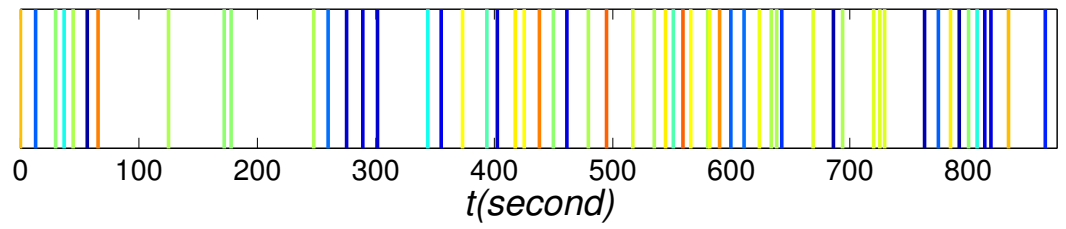

Figure 1: An example of a controller's communication activity in $15 \mathrm{~min}$. Each vertical bar corresponds to a communication event, and different colors represent different flights that the communication was addressing.

\subsection{Temporal network}

We propose a new method for converting controllers' temporal activity data (such as those shown in Figure 1) into undirected, weighted networks. Throughout our analysis in this paper, we assume that

- each instance of the controller's communication is related to a single flight;

- there can be at most one communication made at any point in time.

Both assumptions are verifiable at the sector level. In the following discussions, we focus on a given sector and a time horizon denoted $\left[T_{\min }, T_{\max }\right]$, during which multiple flights may be traversing the sector under the guidance of the air traffic controller.

We begin with the temporal network, which is a time-varying network that expresses the dynamic relationships among various flights using the sector. Traditionally, a network is represented as a graph $G(\mathcal{V}, \mathcal{L})$, where $\mathcal{V}$ and $\mathcal{L}$ are the sets of nodes and links, respectively. In the temporal network, $\mathcal{V}$ contains all the flights traversing the sector within $\left[T_{\min }, T_{\max }\right]$. The links should indicate the relationship between two flights, and must take into account the air traffic controller's involvement. Conventionally, the linkage between two flights is identified through the physical configuration of the flights, for instance the distances that have been used to calculate the traffic complexity (Lacasa et al., 2008). However, such indices have no reference to the traffic controllers. Instead, we propose to link the nodes (flights) using controller's temporal communication data (e.g., see Figure 1), under the premise that the sequence of the communication events reflects, to certain degree, the physical configuration of the airspace and flights involved. For example, two flights have no relationship if all of their communication events are separated by long periods of time. On the other hand, the two flights are related if they are called within a short time span.

Mathematically, we define the following temporal distance between any two flights, $F_{i}$ and $F_{j}$, at time $t \in$ $\left[T_{\min }, T_{\max }\right]$.

$$
D(i, j ; t) \doteq \tau_{j}(t)-\tau_{i}(t)-l_{i}(t), \quad \tau_{j}(t)>\tau_{i}(t)
$$

where given any $t \in\left[T_{\min }, T_{\max }\right], \tau_{i}(t)$ denotes the start time of the latest call event for flight $F_{i}$, and $l_{i}(t)$ is the duration of this call. These new quantities are illustrated in Figure 2.

Before we introduce the temporal network, we first partition the entire time horizon $\left[T_{\min }, T_{\max }\right]$ into several observational periods (termed time window subsequently) of equal size. The reason for this is that a typical flight only stays in the sector for a relatively short time compared to the entire horizon; thus it makes sense to only discuss 


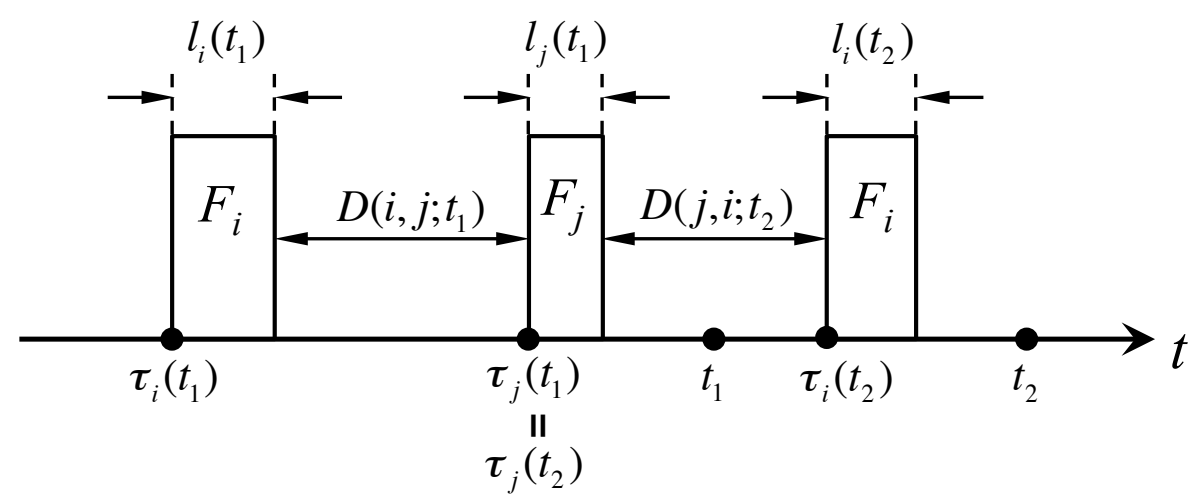

Figure 2: Illustration of the temporal distance function $D(i, j ; t)$.

connections between two flights when they are both present in the sector. For any integer $n \geq 1$, we define intervals $I_{1}, I_{2}, \ldots, I_{n}$ such that the length of each interval is $\tau_{t w} \doteq \frac{T_{\max }-T_{\min }}{n}$. The connectivity among flights are subsequently defined for each time window $I_{k}, k=1, \ldots, n$.

Within each time window $I_{k}$, we use a prescribed time threshold $\tau_{\min }>0$ to determine the connectivity between two nodes (flights). If $D(i, j ; t)$ is smaller than $\tau_{\min }$, then we say that these two flights are related and a link will be established between the corresponding nodes; otherwise the nodes are not connected directly. The following adjacency matrix of the temporal network is consequently obtained.

$$
E(i, j ; t, D) \doteq\left\{\begin{array}{ll}
1 & \text { if } D(i, j ; t)<\tau_{\min } \\
0 & \text { otherwise }
\end{array} \quad \forall i, j \in \mathcal{V}, t \in \tau_{k}, k=1, \ldots, n\right.
$$

In addition, we stipulate that $E(i, i ; t, D) \equiv 0, \forall i \in \mathcal{V}$. Here, we emphasize the time-varying nature of the adjacency matrix $E$, as well as its dependence on the special distance $D(i, j ; t)$, by including $t$ and $D$ in the notation (see (2.2)). Since the adjacency matrix $E$ is time-dependent, so must be the temporal network; and this is incorporated in the notation $G(\mathcal{V}, \mathcal{L} ; t)$. A formal definition of the temporal network is as follows.

Definition 2.1. (Temporal network) The temporal network, denoted $G(\mathcal{V}, \mathcal{L} ; t)$, is a time-varying graph where the node set $\mathcal{V}$ contains all the flights traversing the sector during $\left[T_{\min }, T_{\max }\right]$, and the link set is time-varying and defined via the adjacency matrix (2.2).

The temporal network $G(\mathcal{V}, \mathcal{L} ; t)$ contains much information regarding the connections among flights relative to the controller's activities, and sheds light on the controller's dynamics. However, in a temporal network $G(\mathcal{V}, \mathcal{L} ; t)$, the connection between any pair of nodes $(i, j)$ is time-dependent even within the same time window $I_{k}$; it is entirely possible that while a connection is established for some period of time, it may be gone for some other time intervals. We use Figure 3 to illustrate this possibility. We also use Figure 4 to illustrate the time-varying connectivity between nodes across several time windows (we note that the connectivities in Figure 4 are well defined even if a call event $F_{i}$ spans both $I_{k}$ and $I_{k+1}$ ).

In a static network, the degree of a node is defined to be the number of links incident to it. However, in a time-varying network such as the temporal network, the definition of nodal degrees is not straightforward, and there does not exist widely agreed metric to define time-varying degrees in the literature. In this paper we propose the following definition of nodal degrees for the temporal network. For any flight $i \in \mathcal{V}$, we identify the time windows $I_{i_{1}}, I_{i_{2}}, \ldots, I_{i_{m}}$ during which the flight is within the sector. Within each such time window $I_{i_{k}}$, we say that flights $i$ and $j$ are connected if

$$
D(i, j ; t, D)=1 \text { for some } t \in I_{i_{k}}
$$

Note that it is possible that $i$ and $j$ are connected at some time but disconnected at some other time (see Figure 4); in this case, we consider them connected as long as they were connected at least once in this time window. We denote 


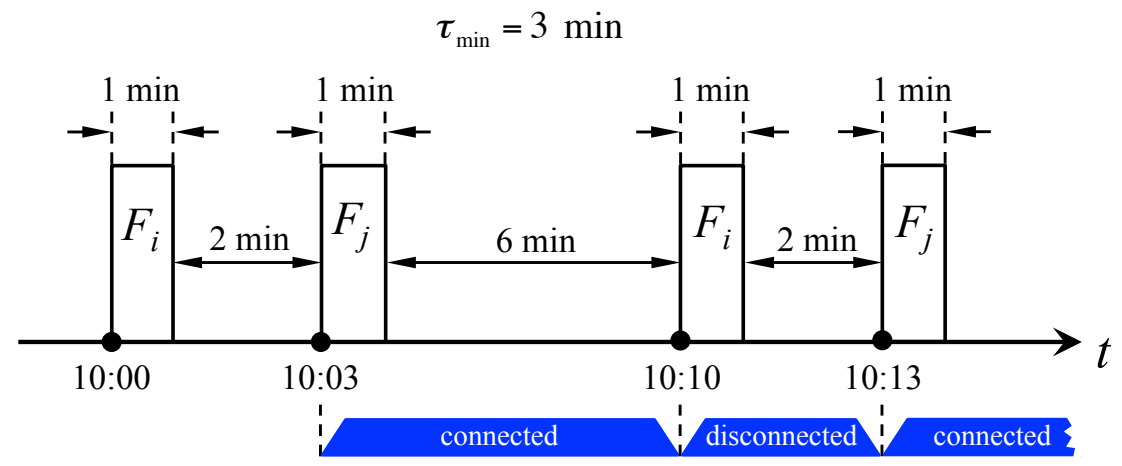

Figure 3: Illustration of the time-varying connectivity between two flights $F_{i}$ and $F_{j}$ within the same time window $I_{k}$. If the threshold $\tau_{m i n}=3$ min, then the connectivity changes with time, as shown in the figure.

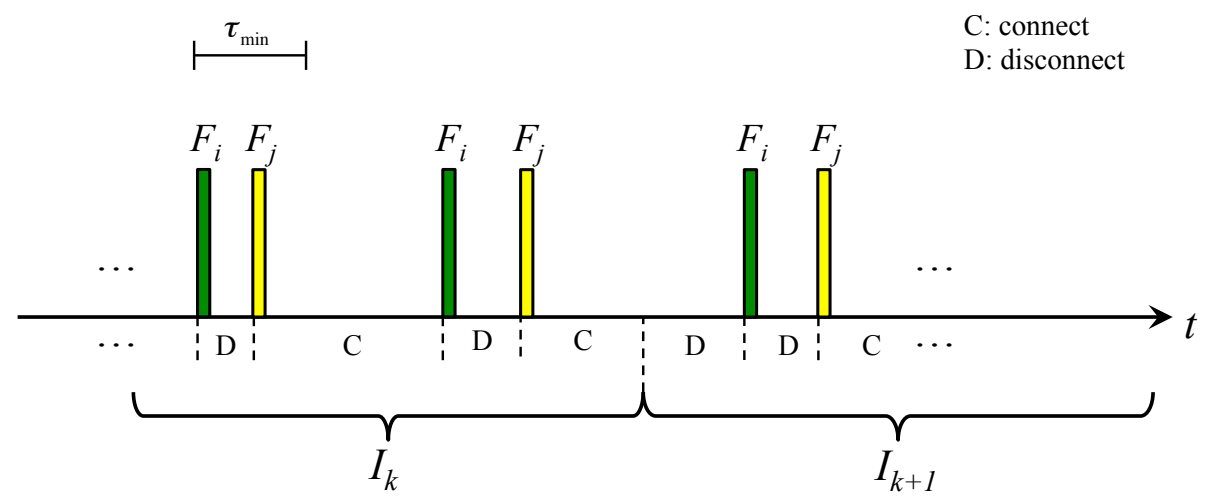

Figure 4: Illustration of the time-varying connectivity across different time windows $I_{k}$ and $I_{k+1}$. See (2.2) for detailed definition of connectivity.

the number of flights connected to $i$ during $I_{i_{k}}$ as $A_{k}^{i}$. Then the vector of time-dependent degrees for flight $i$ is defined as

$$
\mathcal{D}^{i} \doteq\left(A_{k}^{i}: k=1, \ldots, m\right)
$$

As we have seen so far, working with temporal networks can be sometimes cumbersome and lack direct insights. This motivates the time-aggregated network, which represents all these time-varying connections in an efficient and condensed way for the ease of analysis.

\subsection{Time-aggregated network}

Since the links in the temporal network is time-dependent, in order to record and collectively present these timevarying linkage between any pair of nodes, we propose in this section the time-aggregated network, which is a static network including the historical connections between any pair of nodes.

We observe from Figures 3 and 4 that the periods when the connection is present $(E(i, j ; t, D)=1)$ or absent $(E(i, j ; t, D)=0)$ must be disjoint time intervals. This leads to the following definition:

Definition 2.2. (Proper interval) A proper interval is the largest interval such that the connectivity therein is constant (connected/disconnected), and there is at most one call event related to $F_{i}$ or $F_{j}$.

For example, the time intervals $[10: 03,10: 10)$ and $[10: 10,10: 13)$ in Figure 3 are both proper intervals.

Given a pair of flights $F_{i}$ and $F_{j}$, we let $\mathcal{I}_{i, j}^{c}$ be the set of proper intervals when the connection is present; and $\mathcal{I}_{i, j}^{d}$ be the set of proper intervals when the connection is absent, and we denote by $\left|\mathcal{I}_{i, j}^{c}\right|$ and $\left|\mathcal{I}_{i, j}^{d}\right|$ the cardinalities (number of elements) of these sets. In other words, there are, cumulatively, $\left|\mathcal{I}_{i, j}^{c}\right|$ connections between these flights. 
The following straightforward fact is stated without proof; it is useful when we define the length of the links in (2.5).

[Fact]. For any proper interval $\mathcal{I} \in \mathcal{I}_{i, j}^{c} \cup \mathcal{I}_{i, j}^{d}$, the temporal distance $D(i, j ; t)$ is a constant for all $t \in \mathcal{I}$.

We define the symmetric matrix $N=\{N(i, j)\}_{i, j \in \mathcal{V}}$ to record the number of connections between two nodes:

$$
N(i, j)=N(j, i)=\left|\mathcal{I}_{i, j}^{c}\right| \quad \forall i, j \in \mathcal{V}
$$

In addition, for each $\mathcal{I} \in \mathcal{I}_{i, j}^{c}$, we let

$$
L(i, j, \mathcal{I}) \doteq D(i, j ; t) \equiv \text { const. }
$$

be the length of the link corresponding to the proper interval $\mathcal{I}$.

Before we define the time-aggregated network, we need a mechanism for filtering out potential noises in the communication activities. For example, sometimes the controller reads back to the pilots or sends acknowledgement of transmission which, on its own, constitutes a communication event, although the content of that communication is insignificant. Justifiably, we assume that the strength of connection between two flights, $F_{i}$ and $F_{j}$, is positively related to the number of links $N(i, j)$ between them. In other words, the more links there are between $F_{i}$ and $F_{j}$, the stronger their connection is.

Note that in the definition of $E(i, j ; t, D)$ and $\mathcal{I}_{i, j}^{c}$, we have already used the threshold $\tau_{\min }$ to filter out long temporal distances; see (2.2). In adition, we regard the connection between two nodes $F_{i}$ and $F_{j}$ as invalid if the number of links $N(i, j)$ between them is below a threshold $N_{\min }$. Accordingly, the time-aggregated network is defined as follows.

Definition 2.3. (Time-aggregated network) Fix $\tau_{\min }>0$ and integer $N_{\min } \geq 1$. The time-aggregated network, denoted $G\left(\mathcal{V}, \widetilde{\mathcal{L}} ; \tau_{\text {min }}, N_{\text {min }}\right)$, is a static graph whose node set $\mathcal{V}$ contains all the flights traversing the sector during $\left[T_{\min }, T_{\max }\right]$. The adjacency matrix for the network is defined as

$$
A(i, j)=\left\{\begin{array}{ll}
1 & \text { if } N(i, j) \geq N_{\text {min }} \\
0 & \text { if } N(i, j)<N_{\text {min }}
\end{array} \quad \forall F_{i}, F_{j} \in \mathcal{V}\right.
$$

where $N(i, j)$ is given in (2.4).

Here, we provide an example of the time-aggregated network in Figure 5. The left panel of Figure 5 shows the historical communication activities based on calls made by the controller. The colored vertical bars represent call events; the bottom row shows all the calls made within a roughly one-hour period, and the rows above break down the calls by individual flights. The right panel shows the corresponding time-aggregated network obtained from the proposed method. Each node represents a flight, and the size of the node corresponds to the frequency of its communication with the controller. The colors of the nodes indicate different communities identified using the algorithm in Lancichinetti et al. (2011) (see Section 2.4).

The time-aggregated network is parameterized by two constants, $\tau_{\min }$ and $N_{\min }$, which represent the criterion for identifying the relationship between any pair of nodes in the network. $\tau_{\min }$ is concerned with the lengths of the connections between two nodes, and $N_{\min }$ addresses the number of connections. For illustration purpose, in Figure 6 we present time-aggregated networks of the same communication period, with different values of $\tau_{\min }$ and $N_{\min }$. It can be seen that the number of links in a network increases with $\tau_{\min }$ and decreases with $N_{\min }$. From this figure we can also see emerging patterns of connectivity among a group of flights. For example, some nodes have high degrees and are simultaneously connected to several other flights. While the time-aggregated network only presents the connectivity in a cumulative and static way, more time-sensitive properties and characteristics, such as time-dependent degree and network motifs, can be revealed through the temporal network.

Remark 2.4. In this paper, we focus only on the connectivity of the nodes in the network. However, with the help of link length $L(i, j, \mathcal{I})(2.5)$ and number of connections $N(i, j)(2.4)$, one can also calculate the weights of the links between nodes, and analyze the controller dynamics from the perspective of spatial networks. 

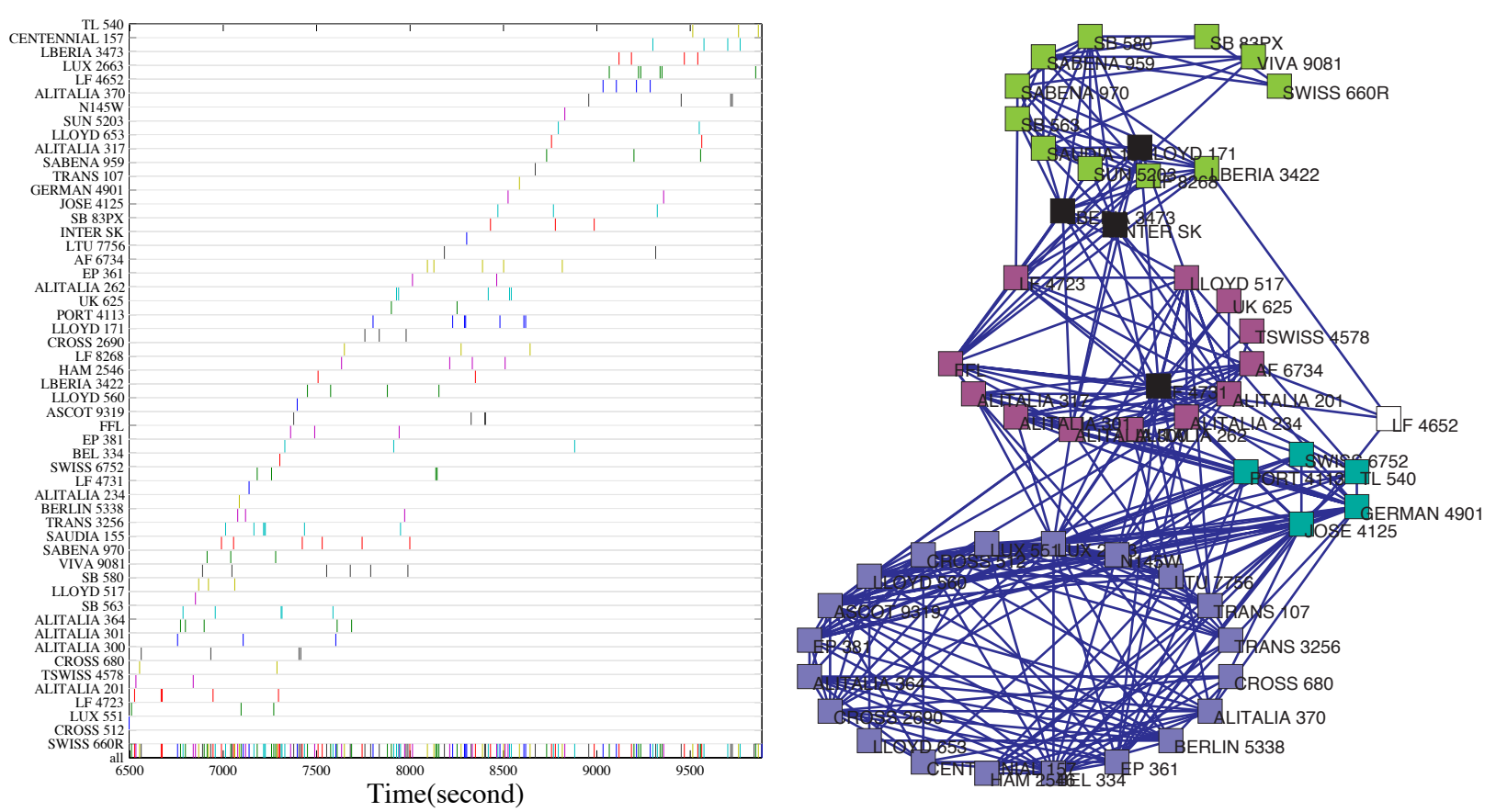

Figure 5: Example of a series of communication events (left) and the associated time-aggregated network (right). On the right panel nodes with the same color belong to the same community.

\subsection{Community detection}

A community is defined, via connectivity, to be a dense sub-network within a larger network. A node is 'more likely' to be linked with the nodes in the same community than others. The community is of particular interest because it may not only correspond to a functional unit of the complex system, but also tell the properties of the individual community structure. Algorithms for detecting communities in complex networks are categorized into three types, namely hierarchical clustering, optimization, and stochastic block model; see Newman (2012) for a review of these methods. Hierarchical clustering assigns nodes to different communities based on the strength of the linkage among the nodes; the optimization methods originate from the equilibrium physical processes; and the stochastic block model is based on the idea of generating networks having community structure with the stochastic establishment of links between the nodes.

Here, we provide a brief introduction to the stochastic block model for community detection, while referring the reader to Newman (2012) and Lancichinetti et al. (2011) for more details. The block model is based on statistical inference by fitting a statistical network with certain community structure to the subject network. We assume the statistical network has $n$ nodes, labeled $\{1,2, \ldots, n\}$, and $m$ communities, $\left\{s_{1}, s_{2}, \ldots, s_{m}\right\}$, such that each node is assigned to exactly one community. Normally, $n$ is the same as the number of nodes in the subject network. Then we define $p_{s_{i}, s_{j}}$ to be the probability that there will be an edge between a node in community $s_{i}$ and a node in community $s_{j}$ in the statistical network; such probabilities are parameters to be determined in the fitting phase of the block model, based on the maximum likelihood principle. More specifically, for any two nodes $i$ and $j$ in the subject network, we let $s_{i}, s_{j}$ be the communities in the statistical network to which $i$ and $j$ belong, respectively. We let $A(i, j)$ be an element of the subject network's adjacency matrix (such as (2.6)). Then the fitting of the statistical model to the subject model amounts to the following maximization problem:

$$
\max _{\substack{p_{s_{i}, s_{j}} \\ 1 \leq i, j \leq n}} L=\prod_{i<j} p_{s_{i} s_{j}}^{A(i, j)}\left(1-p_{s_{i} s_{j}}\right)^{1-A(i, j)}
$$

In this paper, a community refers to a group of flights that can be seen as certain functional component of the traffic evolution. In analogy to the generation of links in the statistical network mentioned above, the communication 

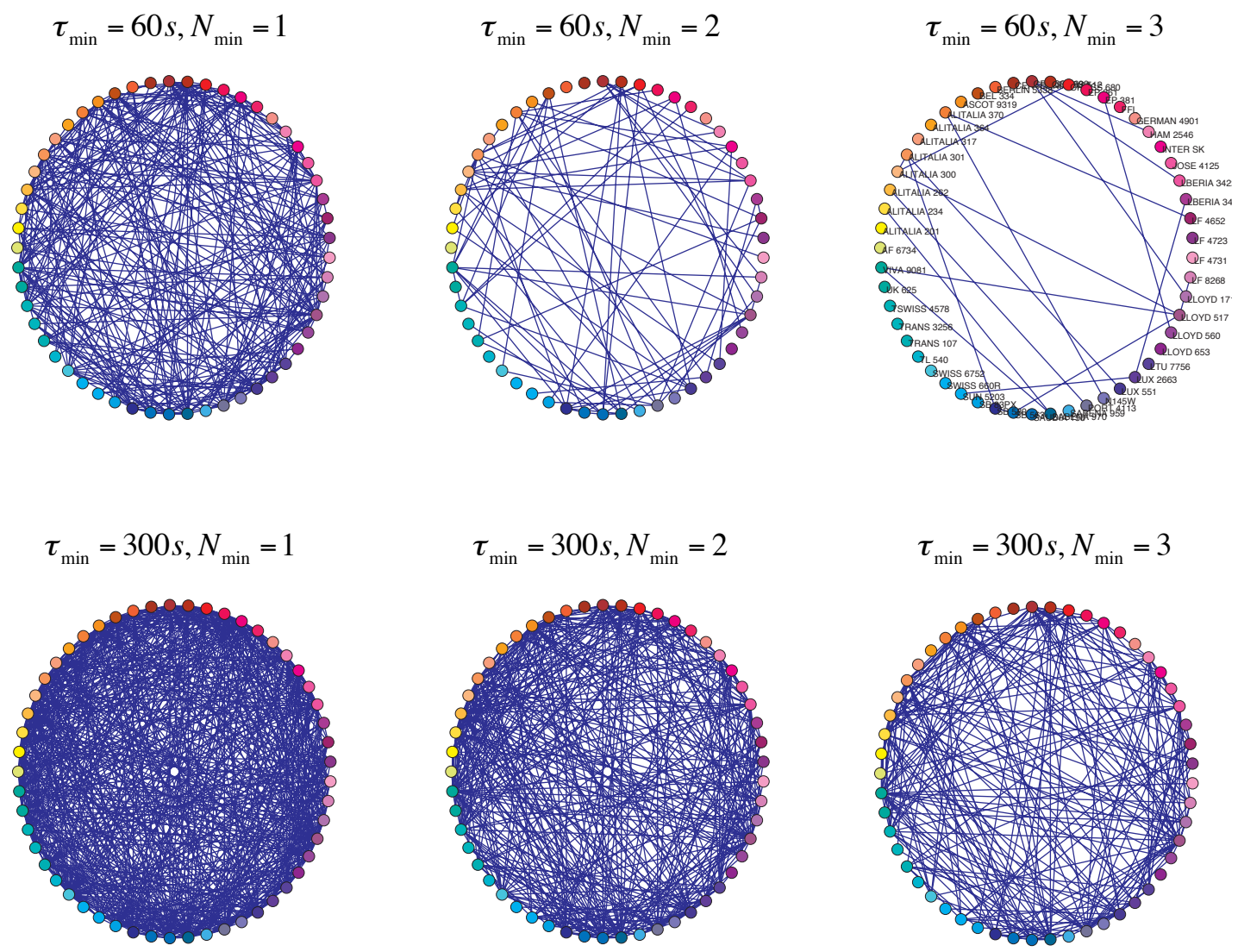

Figure 6: Time-aggregated networks based on the same communication data. The networks differ by the parameters $\tau_{\min }$ and $N_{\min }$.

between the controller and the flights (nodes) can be seen as adding linkage between the flights in the sector. Due to this similarity, the stochastic block model is chosen here to identify the community structure in the time-aggregated network. Detailed algorithm for the block model employed in this paper can be found in Lancichinetti et al. (2011).

The community is defined based on connectivity, which means that it is a topological index. We are the first to link this notion with air traffic operation, which reveals some interesting relationships such as the strong correlation between community size and traffic volume (see Section 4.1.2). The community can be used to provide some useful insights into the inherent structure of the time-aggregated network and, in turn, the dynamics of the controller's activity. For example, it may be used to study the controller's different grouping behaviors when it comes to selecting flights to contact, by providing a quantitative measure based on the network framework that we propose in this paper.

\subsection{Summary and discussion}

So far in this section, we have presented a time-dependent network and a static network, both based on the communication events between the controller and the flights. The definition of these networks relies on critical information regarding the temporal gap between calls made to different flights. The underlying premise of our approach is that calls with temporal proximity are likely to be highly correlated, possibly due to potential conflicts of relevant flights. Therefore, this paper explores the the relationship among flights through a network approach based on time series data. And, we use flexible modeling parameters such as $\tau_{\min }$ and $N_{\min }$ to identify connections between flights and assess the strength of those connections. In such a way, the proposed networks encapsulate much information regarding the flight dynamics and reflect characteristics of the controller's communication activity.

Both the temporal network and the time-aggregated network contain unique information not available in the other. For example, the time-aggregated network contains information regarding the connectivity among flights throughout the study horizon. However, the chronological order of the establishment and disappearance of those connections 
is excluded. Figure 7 shows, on the first three pictures, the temporal network over three short periods. Here, the order in which the links are established has been indicated by the red numbers next to the links. The last picture is a time-aggregation of the first three. Figure 7 reveals that, while the node $F_{7}$ has overall 5 connections with other nodes (see the aggregate network), this number is changing with time and, at any point in time, it is likely to be 2 (see the first three networks). Furthermore, the temporal network also shows the sequence of connections made between flights, which gives rise to certain class of patterns that recur much often, called motifs. For example, the first two networks show chains, the third one shows a loop, and the last one shows star (centered around $F_{7}$ ). These motifs carry much information regarding the process dynamics of the underlying network, and will be analyzed later in detailed in Section 4.2.2.

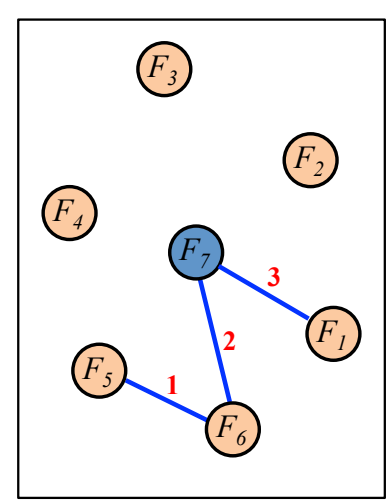

Period I

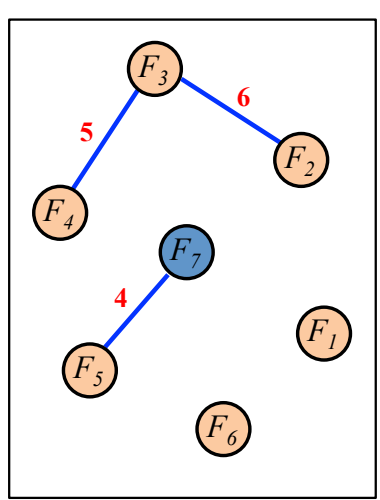

Period II

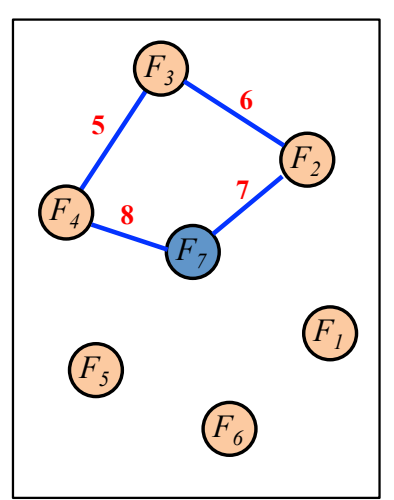

Period III

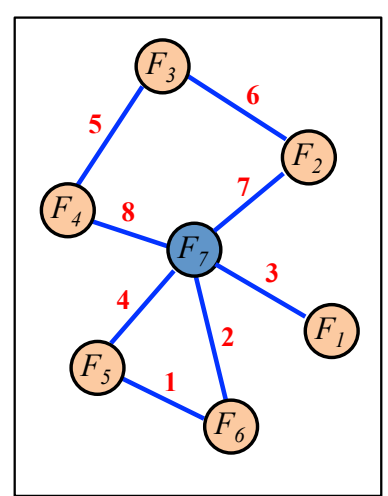

Aggregate

Figure 7: Illustration of the temporal networks (first three networks) and the time-aggregated network (the last network). The nodes $F_{1}-F_{7}$ represent flights, the links represent connections, and the red numbers next to the links indicate the chronological order of their establishments.

\section{Empirical Data}

In this paper, three data sets, $D_{1}, D_{2}, D_{3}$, are considered. $D_{1}$ and $D_{2}$ contain controllers' communication data recorded during human-in-the-loop simulations at EUROCONTROL Experimental Center, and $D_{3}$ is synthetic and constructed based on $D_{2}$. Human-in-the-loop simulation is widely used as an evaluation tool to test new automation procedures by allowing controllers to direct the activities of simulated air traffic while monitoring the effect of the newly implemented procedures, or as a necessary training stage for the novice controllers before controlling realworld traffic

\subsection{Dataset}

$D_{1}$ dataset is provided by the Air Traffic Control Simulation Speech Corpus (ATCOSIM) from EUROCONTROL Experimental Center, and contains ten hours of controller's communication data, recorded during real-time simulations between 20/01/1997 and 14/02/1997 (Hering, 2001). The speech signal data, transcription of the utterance, and annotation and meta-data for all utterance included in the communication data are saved in the database. Specific information on the air traffic and airspace related to the controllers' communication is excluded.

In the $D_{1}$ dataset, there are totally 50 exercises carried out in the simulation; each exercise involves one sector. Table 1 shows some general information of $D_{1}$.

\section{2. $D_{2}$ Dataset}

The $D_{2}$ dataset contains the Paris Terminal Maneuver Area (TMA) simulation data, recorded during a two-week real-time simulation at EUROCONTROL Experimental Center in June 2010. The simulation targeted the French airspace, which covers approach sectors around the Paris CDG airport and surrounding en-route sectors. Around 100 qualified controllers participated in the simulation. The traffic load for the simulation was based on real-world traffic 
Table 1: Information on the 50 exercises in the ATCOSIM database

\begin{tabular}{lrr}
\hline Number of exercises: 50 & Average per \\
Number of sectors per exercise: 1 & Total & $\begin{array}{r}\text { Avercise per sector } \\
\text { ex }\end{array}$ \\
\hline Duration & $39 \mathrm{~h} \mathrm{19min}$ & $1 \mathrm{~h} 11 \mathrm{~min}$ \\
Number of flights & 3121 & 62.42 \\
Number of communication events & 10,078 & 201.56 \\
\hline
\end{tabular}

Table 2: Information on the 14 exercises in the Paris TMA database

\begin{tabular}{|c|c|c|}
\hline $\begin{array}{l}\text { Number of exercises: } 14 \\
\text { Number of sectors per exercise: } 22\end{array}$ & Total & $\begin{array}{r}\text { Average per } \\
\text { exercise per sector }\end{array}$ \\
\hline Duration & $427 \mathrm{~h} 10 \mathrm{~min}$ & 1h $23 \mathrm{~min}$ \\
\hline Number of flights & 17626 & 57 \\
\hline Number of communication events & 55379 & 180 \\
\hline
\end{tabular}

data on 29 May and 12 June, 2009. A total of 14 exercises were carried out in this study, each one with 22 sectors involved. Table 2 shows some basic information related to the Paris TMA dataset.

The data were collected and extracted into three categories as follows.

a. Radio communication data. This type of data only contains the start time epoch and end time epoch for the communication made by the controller or pilots. It has been reported that an average of 300 communication events were made by the controller during each exercise.

b. Pilot maneuver data. This type of data contains the flights' maneuver data as well as the flights' IDs.

c. Transfer information. This type of data contains flight transfer time, i.e. the time epoch when the flight entered/left the sector. It has been detected in the simulation that the throughput of the sector varied from 30 to 100 during the data collection period.

In $D_{2}$, no flight ID information is embedded in the radio communication data, and we reconstruct such information using the pilot maneuver data as follows. We assume that each instance of a flight's maneuver was a result of the controller's call ${ }^{3}$, and that there is a 1-2 seconds delay between the call and the corresponding maneuver. The controller's communication data is constructed thusly. Figure 8 summaries the information on traffic and communication in the dataset.

\section{3. $D_{3}$ Dataset}

The dataset $D_{2}$ is utilized for our investigation by assuming that an instance of aircraft maneuver is a result of the controllers call. However, not every controllers call results in an aircraft maneuver, which means that the way we process $D_{2}$ may underestimate the number of call events (and we cannot rely on the radio communication data in $D_{2}$ alone since it does not contain flight ID information). To remedy this situation, we propose a more sophisticated way to estimate the call events as well as the flight they were addressing; this is done using the following algorithm, which gives rise to dataset $D_{3}$. The time horizon of our analysis is denoted $\left[T_{\min }, T_{\max }\right]$.

\section{Algorithm: Reconstructing communication data}

Step 1 Sort the communication events, transfer data, and pilot's manipulation data in chronological order.

\footnotetext{
${ }^{3}$ This assumption is validated through interviews of the pilots, which suggest that for large or busy terminal airspaces (which is the case for $D_{2}$ ), airplane maneuver performed by the pilots needs to be approved by the controller due to heavy air traffic.
} 


\section{(i) Traffic volume}
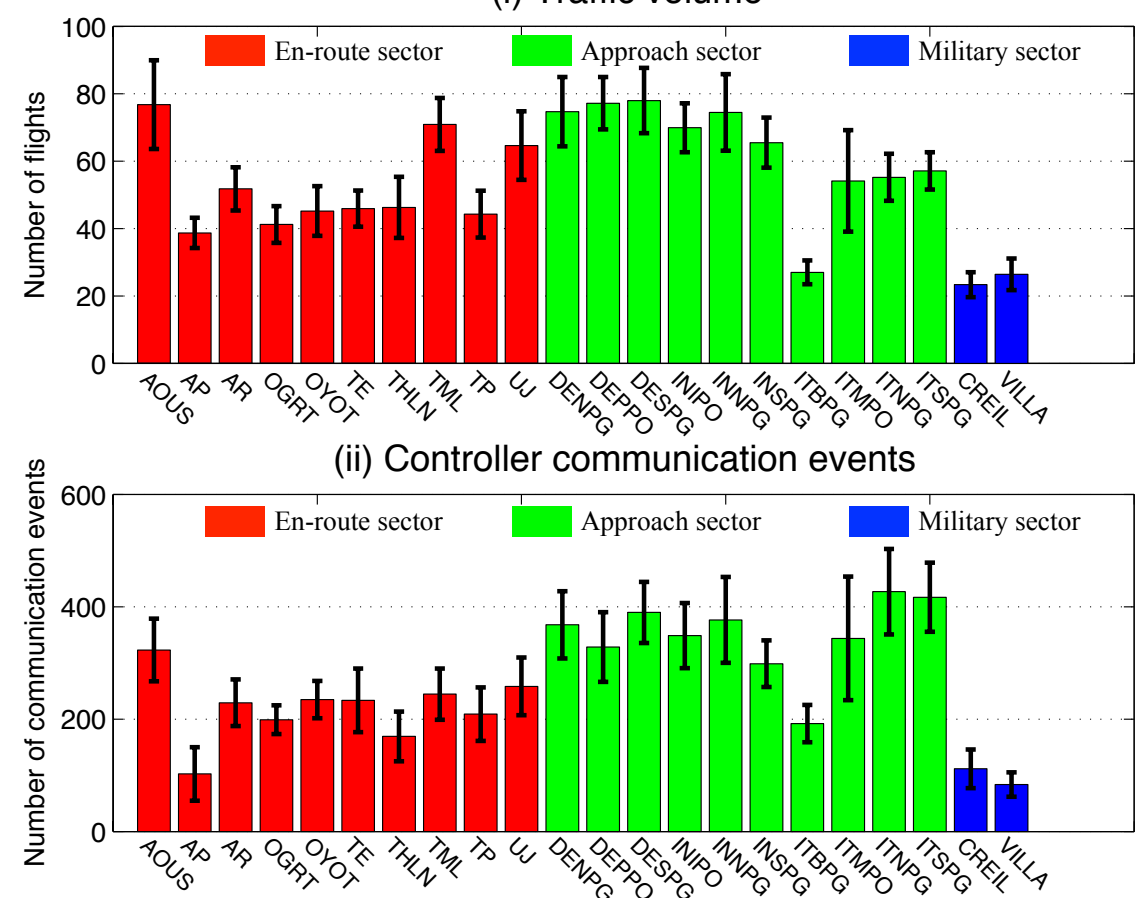

Figure 8: Overview of the traffic and controller communication activities in the Paris TMA data $\left(D_{2}\right)$. The horizontal axes shows different sectors. The heights of the bars indicate the average value in each sector, and the dark vertical segments show the standard deviation across all the exercises.

Step 2 Based on the transfer data, for each instance of the pilot's maneuver, select the nearest controller communication event and match it with the associated flight's callsign. Let $t_{1}=T_{\min }$.

Step 3 If $t_{1} \geq T_{\max }$ or there is no transfer event during $\left[t_{1}, T_{\max }\right]$, then terminate the algorithm. Otherwise, locate the next transfer event $T_{\text {next }}$ in the transfer data, and let $t_{2}=T_{\text {next }}$. Identify all the flights within the sector during time $\left[t_{1}, t_{2}\right]$, and denote the flight set by $F^{t_{1}, t_{2}}$. Let $C^{t_{1}, t_{2}}$ be the set of communication events that are not assigned to any flight callsign during the time $\left[t_{1}, t_{2}\right]$.

Step 4 For each communication event in $C^{t_{1}, t_{2}}$, randomly assign it to a flight in $F^{t_{1}, t_{2}}$. Let $t_{1}=t_{2}$ and go to Step 3.

Remark 3.1. The total number of communication events involved in the random assignment process in Step 4 turns out to constitute less than $1.7 \%$ of all the communication events. Therefore, for our particular dataset the impact of the random selection is rather insignificant, which justifies Step 4.

\section{Numerical Results and Analysis}

In this section, we present the results obtained from the three datasets and provide discussions of our methods and findings. Table 3 presents some general information about the time-aggregated networks constructed by our algorithms.

\subsection{Time-aggregated networks}

We first focus on the static, time-aggregated networks. Our analyses for this type of networks focus on the degree distribution and community structure, and are presented in Sections 4.1.1 and 4.1.2, respectively. 
Table 3: Summary of the time-aggregated networks obtained from the three datasets for different values of $N_{\min }$. The average and standard deviation are over all sectors and all exercises.

\begin{tabular}{llrrrrrr}
\hline & & \multicolumn{2}{c}{$D_{1}$ (ATCOSIM) } & \multicolumn{2}{c}{$D_{2}$ (Paris UM) } & \multicolumn{2}{c}{$D_{3}$ (Paris M) } \\
& & average & std & average & std & average & std \\
\hline \multirow{2}{*}{$N_{\min }=1$} & \# of nodes & $\mathbf{6 2 . 0}$ & $\mathbf{5 . 9}$ & $\mathbf{4 9 . 1}$ & $\mathbf{1 8 . 1}$ & $\mathbf{5 3 . 1}$ & $\mathbf{1 8 . 3}$ \\
& \# of edges & $\mathbf{5 9 7 . 2}$ & $\mathbf{1 5 5 . 0}$ & $\mathbf{1 9 4 . 2}$ & $\mathbf{1 1 7 . 7}$ & $\mathbf{2 7 0 . 8}$ & $\mathbf{1 6 2 . 1}$ \\
\hline$N_{\min }=2$ & \# of nodes & $\mathbf{6 2 . 0}$ & $\mathbf{5 . 9}$ & $\mathbf{4 9 . 2}$ & $\mathbf{1 8 . 0}$ & $\mathbf{5 3 . 1}$ & $\mathbf{1 8 . 3}$ \\
& \# of edges & $\mathbf{3 1 3 . 1}$ & $\mathbf{1 4 1 . 7}$ & $\mathbf{1 3 9 . 0}$ & $\mathbf{9 1 . 8}$ & $\mathbf{2 0 7 . 4}$ & $\mathbf{1 3 0 . 4}$ \\
\hline$N_{\min }=3$ & \# of nodes & $\mathbf{6 2 . 0}$ & $\mathbf{5 . 9}$ & $\mathbf{4 9 . 4}$ & $\mathbf{1 7 . 8}$ & $\mathbf{5 3 . 1}$ & $\mathbf{1 8 . 3}$ \\
& \# of edges & $\mathbf{1 6 8 . 0}$ & $\mathbf{1 0 5 . 0}$ & $\mathbf{9 8 . 9}$ & $\mathbf{7 4 . 1}$ & $\mathbf{1 5 7 . 6}$ & $\mathbf{1 0 6 . 7}$ \\
\hline$N_{\min }=4$ & \# of nodes & $\mathbf{6 2 . 0}$ & $\mathbf{5 . 9}$ & $\mathbf{4 9 . 9}$ & $\mathbf{1 7 . 4}$ & $\mathbf{5 3 . 2}$ & $\mathbf{1 8 . 3}$ \\
& \# of edges & $\mathbf{8 9 . 3}$ & $\mathbf{6 8 . 8}$ & $\mathbf{7 2 . 5}$ & $\mathbf{6 1 . 5}$ & $\mathbf{1 2 0 . 4}$ & $\mathbf{8 8 . 8}$ \\
\hline$N_{\min }=5$ & \# of nodes & $\mathbf{6 2 . 0}$ & $\mathbf{5 . 9}$ & $\mathbf{5 0 . 2}$ & $\mathbf{1 7 . 2}$ & $\mathbf{5 3 . 2}$ & $\mathbf{1 8 . 2}$ \\
& \# of edges & $\mathbf{4 8 . 0}$ & $\mathbf{4 3 . 1}$ & $\mathbf{5 3 . 7}$ & $\mathbf{5 2 . 3}$ & $\mathbf{9 2 . 2}$ & $\mathbf{7 3 . 9}$ \\
\hline \hline & \# of networks (total) & 6233 & & 37875 & & 34971 & \\
\hline
\end{tabular}

\subsubsection{Degree distribution}

One of the most fundamental topological characteristics of the network is degree distribution, which have been considered extensively in studies on network dynamics. The degree, $k_{i}$, of a node (flight) $F_{i}$ is defined as the number of nodes in the network that are connected to it; in other words, it expresses the number of flights related to flight $F_{i}$.

The degree distribution of a network is defined as a discrete probability distribution that expresses the probability of finding a node with degree $k$. Intuitively, if a flight $F_{i}$ enters the sector when there is significant air traffic present in the same sector, $F_{i}$ is likely to be associated with many other flights, and one expects the degree of $F_{i}$ to be high. In order to filer this potential influencing factor, we introduce the normalized degree, $\hat{k}_{i}$, defined as

$$
\hat{k}_{i}=\frac{k_{i}}{N_{\text {traffic }}^{i}},
$$

where $N_{\text {traffic }}^{i}$ is the total number of flights in the sector when $F_{i}$ traverses the sector. Figure 9 plots the normalized degree distribution of flights in the $D_{1}$ dataset.

We have made the following observations based on Figure 9, followed by some discussion.

(a) The shapes of the distributions varies for different $N_{\min }$. As $N_{\min }$ increases, all the distributions (corresponding to different $\tau_{\min }$ ) shift towards left; and the larger $N_{\min }$, the more left-skewed the distributions are. This observation coincides with the definition of $N_{\min }$ as it filters out more links as it gets larger, causing the nodal degrees to drop.

(b) When $N_{\min }$ is fixed, the graph of the distribution shifts towards right as $\tau_{\min }$ increases. This is expected since a higher $\tau_{\min }$ means more strict criterion for identifying a link (see (2.2)).

(c) When $N_{\min }=1$ (i.e. there is no filtering effect from $N_{\min }$; see (2.6)), the distributions, especially for small values of $\tau_{\min }$, follow approximate Poisson distribution (for example, see the first two pictures of Figure 10 for some Poisson fitting results). Poisson or normal degree distributions appear commonly in random networks where each edge is present or absent with equal probability (Erdös and Réyi, 1959). This suggests that the time-aggregated network in this case exhibits the characteristics of a random network, which further implies that the pairs of flights are randomly and uniformly selected by the controller. However, we note that such randomness only exists locally; see Remark 4.1.

(d) For $N_{\min } \geq 3$ the normalized degree distributions become heavily left-skewed and follow approximate powerlaw distribution (e.g. see the third picture of Figure 10). The qualitative change in the distributions from $N_{\min }=1$ to $N_{\min } \geq 3$ suggests a transformation of the network. A reasonable explanation is preferential 

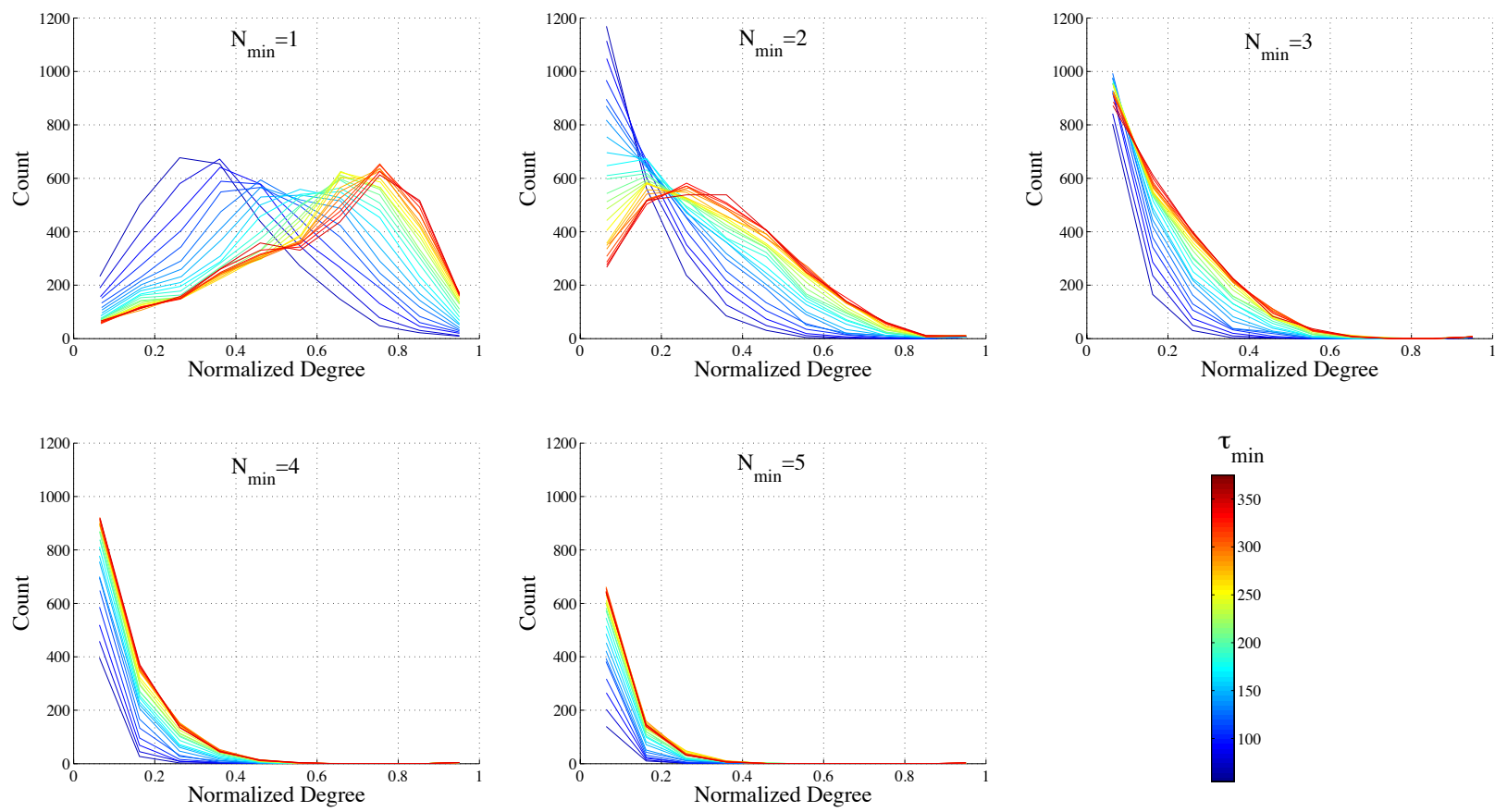

Figure 9: Normalized degree distribution in $D_{1}$. The colors of the lines correspond to different values of $\tau_{\min }$, and are indicated in the color bar.

attachment proposed by Barabási in the explanation of power-law distribution of degree in complex networks (Barabási, 2002). The shift of the network from being random to being "scale-free" as we filter out noises by increasing $N_{\min }$ suggest that the controllers keep a few "important flights" in the call list, and frequently contact them while attending to other flights randomly at the same time.

We note that, however, not all the distributions of the normalized degrees follow Poisson/Normal or power-law distributions. The fitting results in Figure 10 are only illustrative of some distributions, and the key point is that the distributions undergo a qualitative transition as shown in Figure 9.
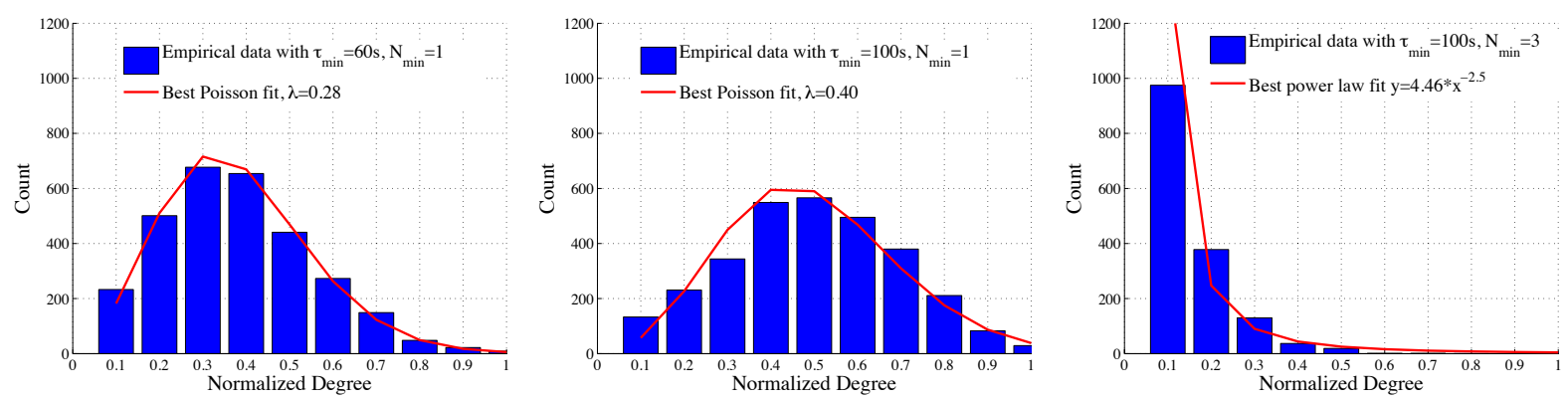

Figure 10: Fitting results for the normalized degree distributions.

Remark 4.1. Distinction needs to be made between the global and local randomness of the communication events. On the global scale, the connections are relatively deterministic since flights enter the sector in a chronological order. For example, a flight in the sector can never have a connection with one that has left the sector 30 min ago. In other words, connections occur only among flights that are close to each other temporally. On the other hand, the local connections for a group of flights with temporal proximity, as our results above suggest, exhibit certain degree of randomness. In summary, the communication events are globally orderly, but locally random. 
Furthermore, the local randomness is partially caused by some call events without substantial information. For example, the controller may need to read back to the pilots who ask for clearance, or provide acknowledgement to the flights' status updates. These kinds of communication events are not part of the systematic scanning technique of the controller, and add to the randomness of the actual call events. Further investigation is needed to closely examine the contents of the calls in order to filter the noises and extract more insights into the dynamics of the controller's communication activity, which will be pursued as a future study with more refined data.

$\mathrm{D}_{1}$
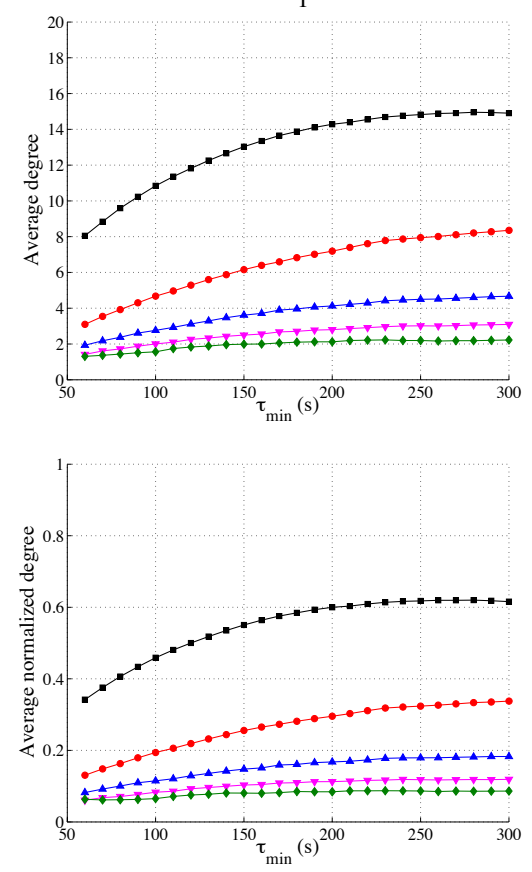

$\mathrm{D}_{2}$
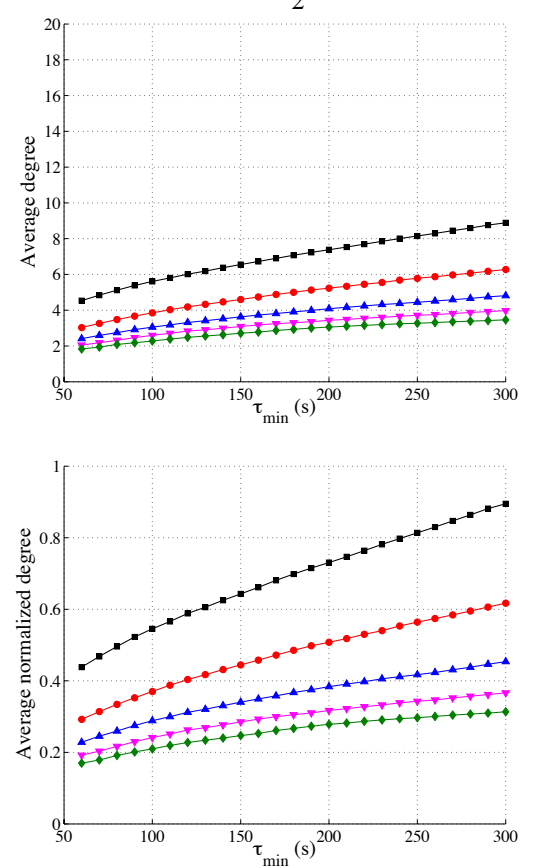

$\mathrm{D}_{3}$
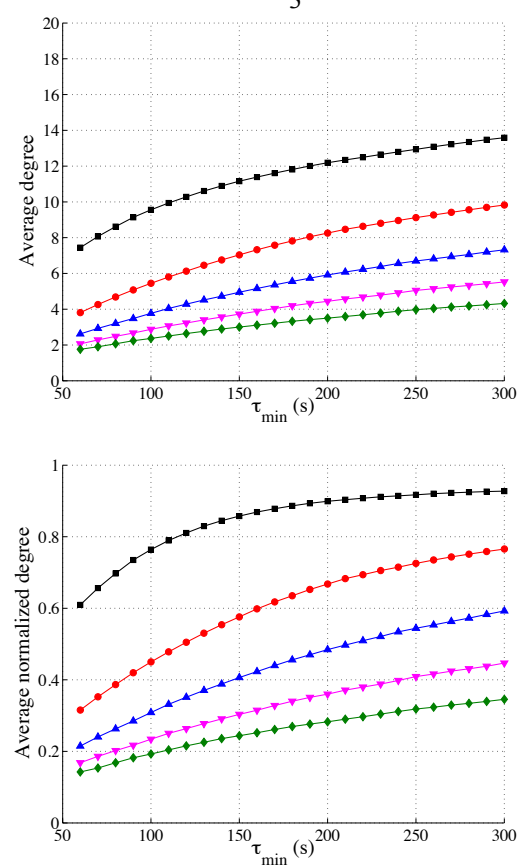

$$
\neg \mathrm{N}_{\text {min }}=1 \rightarrow \mathrm{N}_{\text {min }}=2 \neg \mathrm{N}_{\text {min }}=3 \rightarrow \mathrm{N}_{\text {min }}=4 \rightarrow \mathrm{N}_{\text {min }}=5
$$

Figure 11: Effects of $N_{\min }$ and $\tau_{\min }$ on the average degree. First row: average degree; second row: average normalized degree.

In order to reveal further information on the network structure and the operational characteristics, we show in Figure 11 the average (normalized) degrees of the nodes in the network. Again, we make the following key observations.

(e) The averaged (normalized) degree always increases with $\tau_{\min }$ and decreases with $N_{\min }$, which coincides with their definition.

(f) For all three datasets, $D_{1}, D_{2}$ and $D_{3}$, the gap between the curves corresponding to $N_{\min }=1$ and $N_{\min }=2$, and the gap between the curves corresponding to $N_{\min }=2$ and $N_{\min }=3$, far exceed those between other curves. This indicates that regardless of the choice of $\tau_{\min }$, we have $N(i, j)=1$ or 2 for most of the node pairs $(i, j)$. In other words, historically, most pairs of flights have one or two connections. A possible explanation is that most of the flights are flying without the controller's extra intervention. For example, most of the flights in the en-route sector receive one transfer-in message and one transfer-out message if they do not need to change the speed or altitude.

(g) $D_{1}$ (EUROCONTROL ATCOSIM) has the highest degree numbers overall (see the first row of Figure 11), indicating that there are more communication events in this dataset than $D_{2}$ and $D_{3}$, which come from the same source (Paris TMA). However, the situation with the normalized degree (4.7) seems reversed: $D_{2}$ and $D_{3}$ have higher overall normalized degree than $D_{1}$. This means that the high connectivity among flights in $D_{1}$ is most 
likely to be caused by the heavy air traffic in the sector, which serves as the denominator in the definition of normalized degree. On the other hand, although $D_{2}$ and $D_{3}$ encounter considerably lower air traffic (this is apparent from Tables 1 and 2), the number of connections per flight turns out to be higher than $D_{1}$, which means that, for $D_{2}$ and $D_{3}$, on average each flight receives more attention from the controller, despite the lower air traffic flow.

(h) The average (normalized) degrees in $D_{1}$ remain relatively unchanged for $\tau_{\min } \geq 200 \mathrm{~s}$, whereas they continue to increase beyond $\tau_{\min }=200 \mathrm{~s}$ in $D_{2}$ and $D_{3}$. This means that in the Paris dataset $\left(D_{2} \& D_{3}\right)$, a non-negligible amount of flights have temporal distances larger than 200s, while the majority of the flights' temporal distances in the ATCOSIM dataset $\left(D_{1}\right)$ are within 200s. This further highlights the difference in the controllers' dynamics in these two situations: The communication events in the Paris dataset are more sparse in time than the ATCOSIM dataset, possibly due to the lower traffic.

\subsubsection{Correlations between network community and air traffic}

It is of great interest to infer the dynamic information of air traffic from the controller's communication data. The physical configuration of air traffic in the sector is manifested in the controller's mind with certain structure or pattern, and hence reflected in his/her communication events. In this section, we relate the call events to the air traffic profile, by applying community detection techniques to the time-aggregated network. By definition, nodes in the same community have more connections with each other than with other nodes. It is expected that flights in the same community are likely to form a "platoon" that traverses the sector, and thus the average size of communities is related to the air traffic volume in the sector.

Community detection is carried out according to Lancichinetti et al. (2011) based on the adjacency matrix $A$ defined in (2.6). The correlation coefficients and $p$-values are provided in Table 4 for each sector, where the correlation analysis is based on experimental data over all exercises. Note that $p$-values below the confidence level $\alpha=0.025$ indicate high correlation. We found that the correlation between the average community size and the number of flights depends on the sector type; see Figure 8 for a reference of the sector types. In particular, 9 out of the 10 approach sectors are found to have high correlation, while the correlation results are mixed in the other two types of sectors. It is likely that, due to the relatively high traffic flow and intense pilot maneuvers in the approach sectors (mostly near airports), the communication activities are more conflict-driven than random. Therefore, higher traffic volume implies more conflicts at the approach sectors, which in turn implies more frequent communication among the same group of flights, which are more likely to be identified as the same community due to the dense communication. This explains the high correlation between the community structure and the traffic flow at the approach sectors.

Table 4: Correlation between average community size and number of flights in the sector. Sectors with $p$-values below the confidence level 0.025 are highlighted, indicating strong correlation.

\begin{tabular}{rrrrrrrrrrrr}
\hline Sector & AOUS & AP & AR & CREIL & DENPG & DEPPO & DESPG & INIPO & INNPG & INSPG & ITBPG \\
\hline Corrcoef & 0.1063 & $\mathbf{0 . 9 6 5 3}$ & 0.4314 & $\mathbf{0 . 9 2 7 2}$ & $\mathbf{0 . 7 9 7 1}$ & $\mathbf{0 . 7 4 4 3}$ & 0.4240 & $\mathbf{0 . 6 6 7 0}$ & $\mathbf{0 . 5 9 5 4}$ & 0.4672 & $\mathbf{0 . 9 8 1 1}$ \\
$p$-value & 0.7175 & $\mathbf{0 . 0 0 0 0}$ & 0.1235 & $\mathbf{0 . 0 0 0 0}$ & $\mathbf{0 . 0 0 0 6}$ & $\mathbf{0 . 0 0 2 3}$ & 0.1308 & $\mathbf{0 . 0 0 9 2}$ & $\mathbf{0 . 0 2 4 7}$ & 0.0921 & $\mathbf{0 . 0 0 0 0}$ \\
\hline Sectors & ITMPO & ITNPG & ITSPG & OGRT & OYOT & TE & THLN & TML & TP & UJ & VILLA \\
\hline Corrcoef & $\mathbf{0 . 9 4 5 1}$ & $\mathbf{0 . 9 9 2 4}$ & $\mathbf{0 . 9 8 7 1}$ & 0.5729 & 0.5769 & 0.4568 & $\mathbf{0 . 7 9 1 9}$ & $\mathbf{0 . 8 1 9 4}$ & $\mathbf{0 . 6 6 8 1}$ & $\mathbf{0 . 8 6 4 3}$ & $\mathbf{0 . 7 2 6 8}$ \\
$p$-value & $\mathbf{0 . 0 0 0 0}$ & $\mathbf{0 . 0 0 0 0}$ & $\mathbf{0 . 0 0 0 0}$ & 0.0323 & 0.0308 & 0.1006 & $\mathbf{0 . 0 0 0 7}$ & $\mathbf{0 . 0 0 0 3}$ & $\mathbf{0 . 0 0 9 0}$ & $\mathbf{0 . 0 0 0 1}$ & $\mathbf{0 . 0 0 3 2}$ \\
\hline
\end{tabular}

While the analysis of time-aggregated network reveal certain general characteristics of how the controller communicates with the flights, it does not capture the temporal dimension of the communication patterns. Next, we use temporal networks to further investigate the dynamic behavior of the controller.

\subsection{Temporal networks}

The construction of the temporal network relies on the quantity $E(i, j ; t, D)$, which is obtained from the controller's communication data; see (2.2). The network is also parameterized by $\tau_{t w}$, which is the length of the subintervals used to partition the time horizon $\left[T_{\min }, T_{\max }\right.$; ; see Section 2.2. Notice that $\tau_{t w}$ can be seen as the time resolution of the temporal network. In this section, we analyze the temporal network with $\tau_{t w}$ ranging from $60 \mathrm{~s}$ to $300 \mathrm{~s}$. In 
particular, we focus on the time-dependent degree and motifs of the temporal network, which unmasks interesting results regarding the temporal dynamics of the controller's communication activity.

\subsubsection{Time-dependent degree distribution}

The time-dependent degree of a node is computed according to the procedure described at the end of Section 2.2. In Figure 12, we show the time-dependent degrees of all the flights associated with one sector in one exercise in the $D_{1}$ dataset. Note that the degree of a flight is automatically set to be zero if that flight is not within the sector. The upper figure employs $\left(\tau_{t w}, \tau_{\min }\right)=(60,60) \mathrm{s}$, and the lower figure employs $\left(\tau_{t w}, \tau_{\min }\right)=(300,300) \mathrm{s}$. Figure 12 is an example, and the situations with other sectors or datasets are qualitatively similar. The following observations are made based on Figure 12

(i) When $\left(\tau_{t w}, \tau_{\min }\right)=(60,60) \mathrm{s}$, many flights have non-zero degrees for more than one time window. When $\tau_{t w}$ and $\tau_{\min }$ increases to $300 \mathrm{~s}$, most flights have non-zero degrees in just one time window. This is in line with the definition of time window and the way in which the temporal networks are constructed and represented.

(j) For both cases, the majority of the nodes have degrees of 1 or 2 . These nodes are shown as dark blue, and are likely to be less important in the controller's activity.

(k) There are a few clusters in the $\tau_{t w}=60 \mathrm{~s}$ case (highlighted with circles) that have uniformly higher degrees. These areas correspond to relatively intense communication events, and the controller is likely to jump among these flights in the form of chains or loops. When $\tau_{t w}=300 \mathrm{~s}$, most of these clusters disappear, replaced by nodes with very high degrees (indicated as red or orange). This means that, as the observation window $\left(\tau_{t w}\right)$ gets larger, more connections are established in the same time window and, as a result, the chains and loops tend to form stars (see Figure 7); this is also confirmed by Figure 15 later). The nodes with very large degrees are likely to be the center of the controller's attention (star).

Studies on the controller's cognitive activities have found that the airspace structure plays an important role during the controller's interaction with traffic (Histon and Hansman Jr, 2008). To test this hypothesis, we calculate the empirical distribution of time-dependent degrees of each sector. Figure 13 shows the empirical distribution of the time-dependent nodal degrees in the dataset $D_{2}$ (the results for $D_{1}$ and $D_{3}$ are qualitatively similar and thus are omitted here). Each picture in Figure 13 corresponds to a specific value of $\tau_{\min }$, which varies from $20 \mathrm{~s}$ to $180 \mathrm{~s}$, and $\tau_{t w}$ is set to be $180 s$ throughout. Each curve represents the empirical distribution of nodal degrees associated with the same sector, and is obtained by summarizing the degrees over all time windows and all exercises.

Surprisingly, the probability distributions of the time-varying nodal degrees have quite similar shapes across different sectors, which suggests that the airspace structure has little effects on the communication patterns of the controllers. Moreover, when $\tau_{\min }=20 \mathrm{~s}$ the majority (ranging from $40 \%$ to $70 \%$ ) of the nodes have zero degree; when $\tau_{\min }=40 \mathrm{~s}$ the number of zero-degree nodes decreases significantly. This implies that a significant number of node pairs have a temporal distance between 20-40s. Furthermore, for $\tau_{\min } \geq 20 \mathrm{~s}$ most of the nodes have degrees between 0 and 3 , meaning that during the 180 s time window most flights are connected to 0-3 other flights.

We study the coupling effect of $\tau_{\min }$ and $\tau_{t w}$ on the average degree in Figure 14, where the average nodal degrees shown therein are obtained from the empirical distributions in Figure 13. Based on these contour lines we see that the average degree in the temporal network, as a function of $\tau_{\min }$ and $\tau_{t w}$, is continuous, convex, and increasing in both arguments. Moreover, when $\tau_{\min }$ and $\tau_{t w}$ are relatively high, the function is more sensitive to the increase in the other argument, as can be seen from the nearly horizontal (vertical) contour lines near large values of $\tau_{\min }\left(\tau_{t w}\right)$. This is understandable given that when the threshold $\tau_{\min }$ is high, increasing the width of the time windows increases the chance of connections between flights within one time window, and thus the number of connections per flight per time window will increase significantly. On the other hand, when the time window is sufficiently large, increasing $\tau_{\min }$ results in a more relaxed criterion for establishing connections, which also leads to drastic increase in the degrees. Finally, we see that the degrees in the dataset $D_{2}$ are overall much lower than those in $D_{1}$ and $D_{3}$. This is because the way $D_{2}$ is constructed tends to underestimate the number of communication events, resulting in lower connections overall; see the beginning of Section 3.3 for a detailed explanation.

In order to further reveal the pattern of their connections, we shall investigate the network motifs in the next section. 

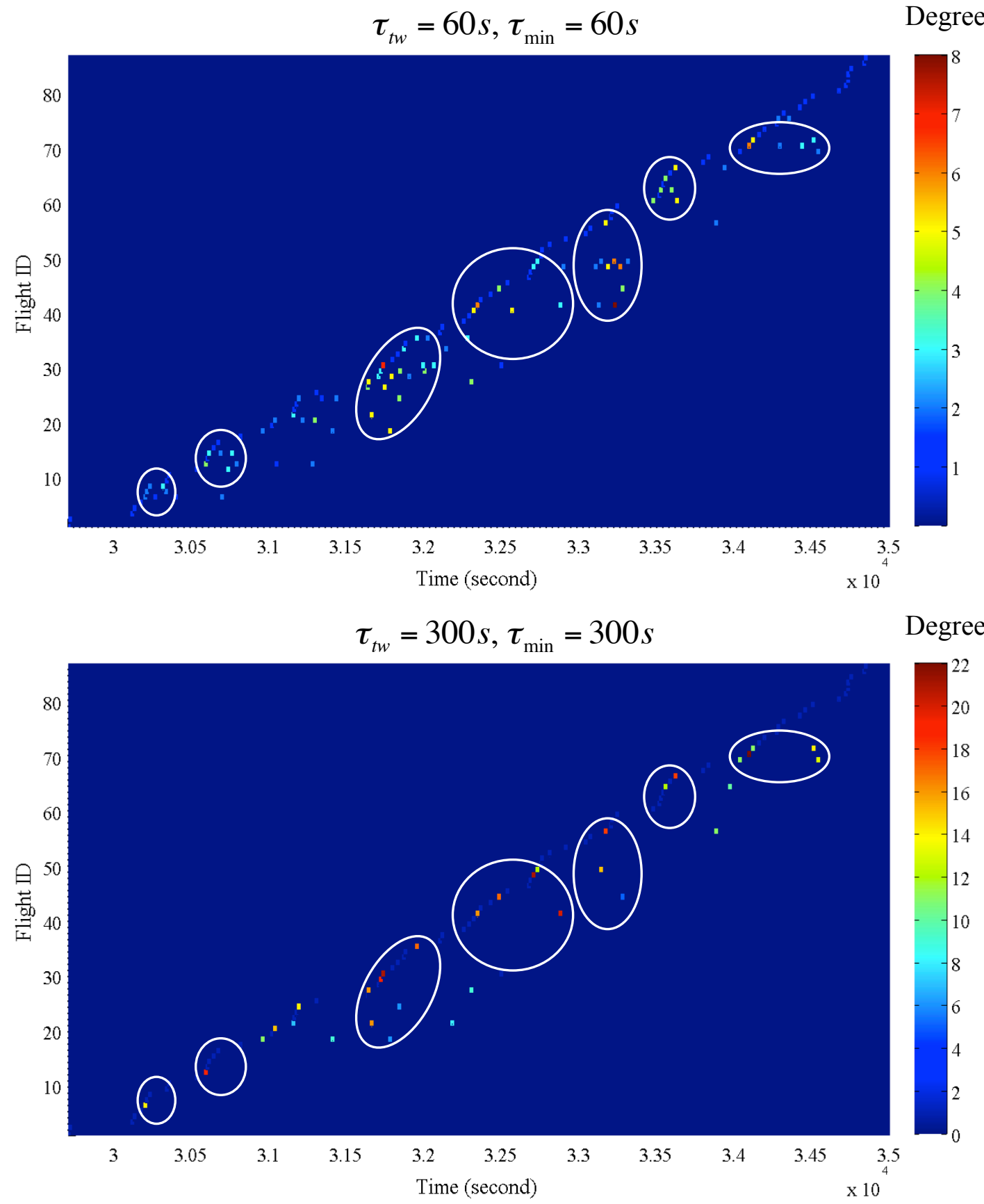

Figure 12: Time-dependent degrees of flights in the $D_{1}$ dataset. The upper figure is based on $\tau_{t w}=60 \mathrm{~s}$; the lower figure is based on $\tau_{t w}=300 \mathrm{~s}$. The flights are labeled according to their entering times.

\subsubsection{Motifs}

A network motif is the equivalence class of subgraphs or patterns that recur much more often than randomly. It has been widely agreed that motifs are closely related to the functionality of the system, and that the detection of network motifs will uncover the information process that are carried out in the network. It is most likely that the air traffic controller allocates his/her attention to the flights according to their dynamic position in the sector. Therefore, 

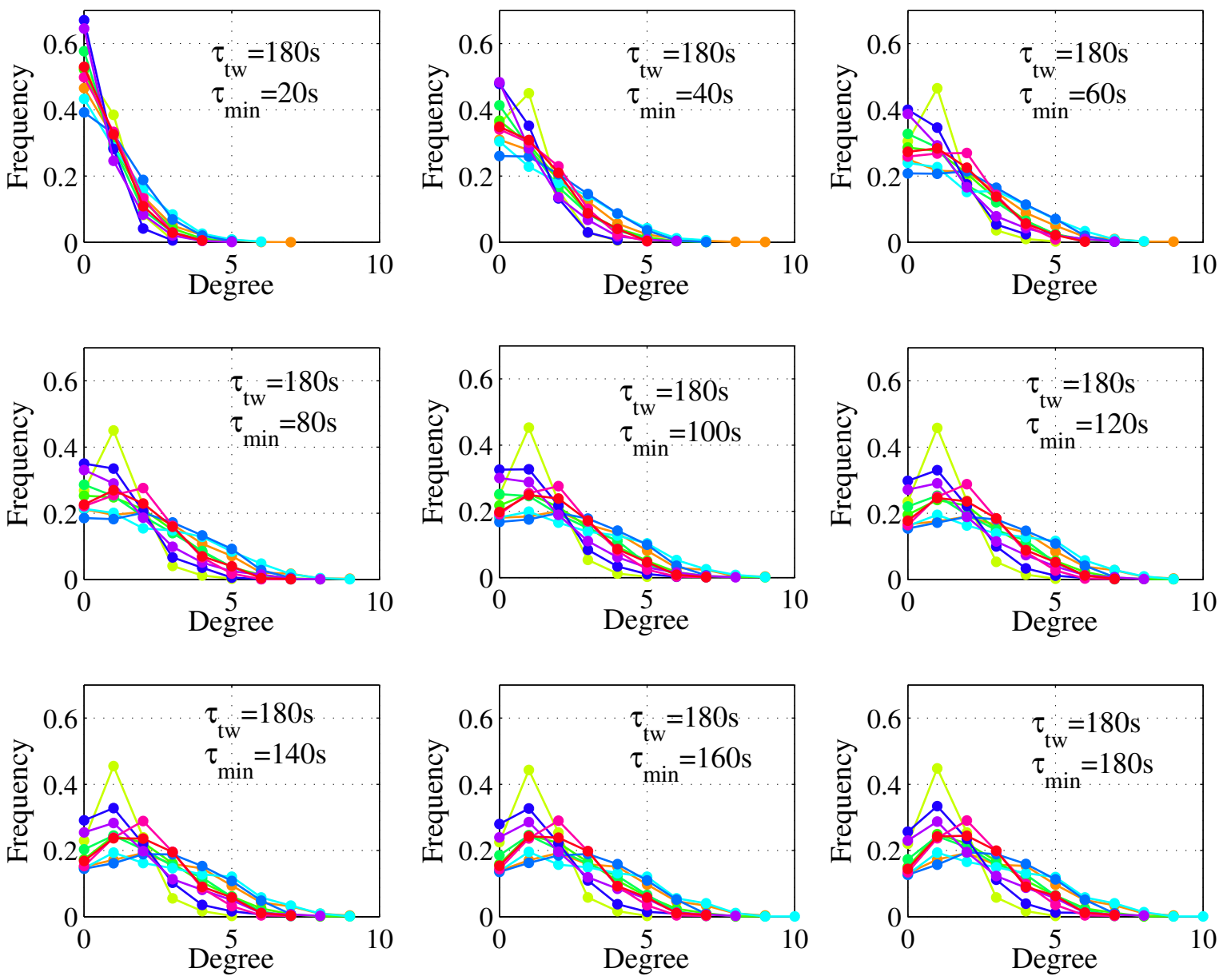

Figure 13: Distribution of time-dependent degrees in the $D_{2}$ dataset. Each picture corresponds to a specific choice of the time window $\tau_{t w}$, and each color (curve) represents a single sector over all the exercises and time windows.

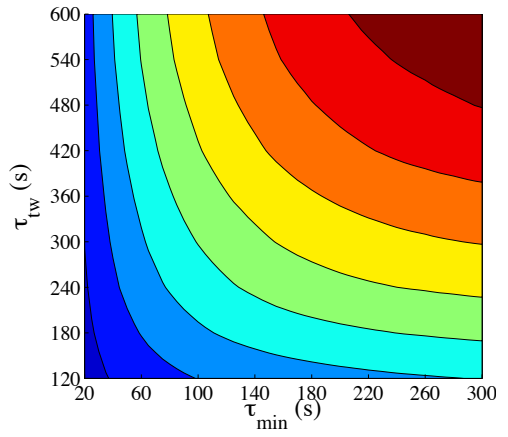

$\mathrm{D}_{1}$

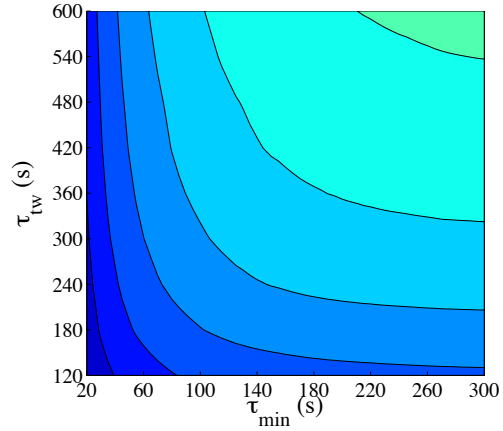

$\mathrm{D}_{2}$

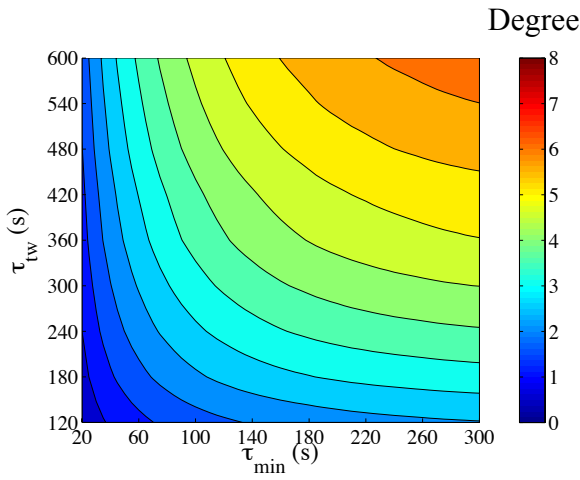

$\mathrm{D}_{3}$

Figure 14: The coupling effect of $\tau_{\min }$ and $\tau_{t w}$ on the average nodal degree. 
airspace configurations such as route structure and dynamic traffic flow will influence the controller's communication patterns and hence be reflected in the network motifs. Our investigation of the temporal network motifs is the first step towards the characterization of the controller's dynamic communication pattern.

Given the fact that the maximum number of flights in the sector is limited, we define the following three basic motifs used in the study of social communication (Zhao et al., 2010). The three types of motifs are (i) Chain: to communicate with different flights in sequence; (ii) Loop: to call the fist flight after calling a number of flights in sequence; (iii) Star: to have a central flight to be constantly called among other less important flights. Figure 7 shows the three types of motifs: The first and second pictures show chains, the third shows loop, and the last one shows star (node $F_{7}$ ).

The three motifs are identified from the temporal networks associated with all the sectors included in our empirical dataset. Notice that the temporal networks are time-discretized by following the procedure described in Section 4.2. Here, we use $\tau_{t w}=\tau_{\min }$ as the free parameters, and calculate the frequencies of the three motifs detected in the discrete temporal network as functions of $\tau_{t w}$, which are shown in Figure 15. We make the following key observations:

(1) When $\tau_{t w}$ is small, the chains are the most commonly seen communication pattern; with the increase of $\tau_{t w}$, both the loops and stars grow. This is understandable since the longer the observation period $\tau_{t w}$, the more connections there are, and thus the more likely that chains form loops or stars. This is in agreement with Figure 12. In addition, the observation that chains and loops are the most common motifs in the temporal networks has been confirmed in other social communication contexts (Zhao et al., 2010).

(m) As $\tau_{t w}$ gets larger, the increase of loops halts for $\tau_{t w} \geq 200 \mathrm{~s}$ while the number of stars continues to grow. This means that most loops are formed within a time span of 200s, and if the controller visits a flight twice with more than 200s apart, then this flight is most likely to be the center of a star.

(n) The motifs in all three datasets, $D_{1}, D_{2}$, and $D_{3}$, follow qualitatively similar trend, which reveals another regular pattern in the controller's communication process.

Moreover, it can been seen that the chain \& loop curves in all three datasets intersect for $\tau_{t w} \in[150,200]$. Compared to the loop motifs, star motifs grow much slower. Even if the observation time window reaches five minutes, the percentages of star motifs are less than $20 \%$.

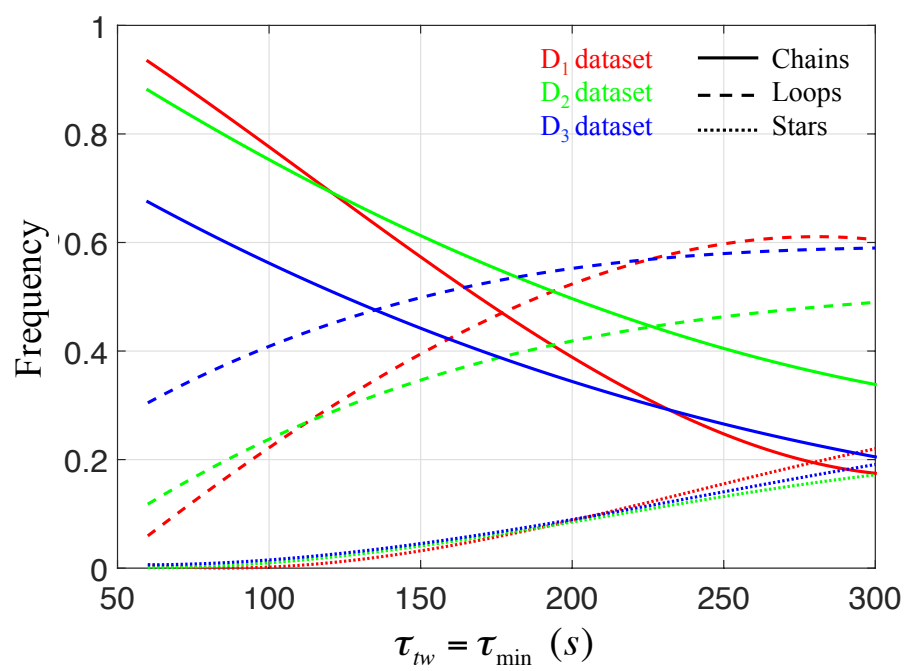

Figure 15: Percentage of three types of motifs detected in the three datasets. Solid lines: Chains; dashed lines: Loops; dotted lines: Stars. Color indicates different datasets $\left(D_{1}\right.$ : red; $D_{2}$ : green; $D_{3}$ : blue). All the curves are the poly fittings to the experimental data. 


\section{Conclusion}

A network perspective is extremely useful for understanding the information diffusion processes in most complex systems. In this paper we have presented a novel network approach for analyzing air traffic controllers' communication activities, which yields new and insightful findings regarding regular patterns and unique characteristics of the controller's communication activities.

The construction of the networks rely on the notion of temporal distance (2.1), which measures the relationship between two flights. The temporal network is then constructed with its node set being the set of flights traversing the sector, and the links defined by comparing the temporal distances with a given threshold. The temporal network is time-varying since the linkage between two flights may change over time. In order to represent the dynamics in a condensed manner, we introduce the time-aggregated network by taking into account all the historical connections between two flights.

We conduct extensive analysis on the proposed networks, in terms of degree distribution, community structure, and motifs. Our findings have identified regular patterns of the controllers' activities across all sectors and repetition of exercises, which suggest universal properties of the controller's activity dynamics. In particular, our analysis on the degree distribution provides evidence that the controller's call events are locally random. However, the actual call events may contain lots of random 'noises' in the form of readbacks and acknowledgements, which do not contain useful information regarding the dynamics of the flights and the controller, and need to be filtered properly to gain more insights into the behavioral patterns of the controller. The correlation between the sizes of network communities and traffic volume reveal that the proposed communication network preserves certain traffic information. Our observation made on the motifs of the networks yields, in a quantitative way, universal patterns of the change of the three motifs considered as the observational period changes.

The proposed framework does not consider the specific airspace configuration or structure, which may lead to the loss of certain information, such as the boundary effect when flights are close to key areas such as sector boundary and intersection points, which may have a considerable effect on the communication as controllers provide clearance or special instructions to the flights. These additional spatial characteristics will be taken into account in a future research.

Although our proposed network approach based solely on the controller's voice communication data is able to identify the general information flow patterns, applying airspace route structure information could further assist the investigation of the information diffusion process, since much of the network structure is manifested through the information dynamics. Local dynamics of the controller's communication is a subject worthy of further investigation as it contains information regarding the microscopic dynamic of the controller's attention. The significance of this paper is that it brings network science into the analysis of the controller's communication dynamics, allowing the former to leverage its findings, emerging techniques, and open questions to facilitate the understanding of the latter.

\section{Acknowledgments}

This research was partially supported by the National Natural Science Foundation of China (Grant Nos. 61304190, 11175086), the Fundamental Research Funds for the Central Universities (Grant No. NJ20150030), and the Natural Science Foundation of Jiangsu Province of China (Grant No. BK20130818).

\section{References}

Ahmed, A. and Xing, E. P. (2009). Recovering time-varying networks of dependencies in social and biological studies. Proceedings of the National Academy of Sciences, 106(29):11878-11883.

Alam, S., Lokan, C., Aldis, G., Barry, S., Butcher, R., and Abbass, H. (2013). Systemic identification of airspace collision risk tipping points using an evolutionary multi-objective scenario-based methodology. Transportation Research Part C: Emerging Technologies, 35:57 - 84.

Averty, P., Collet, C., Dittmar, A., Athenes, S., and Vernet-Maury, E. (2004). Mental workload in air traffic control - an index constructed from field tests. Aviation, Space, and Environmental Medicine., Vol. 75, no. 4, pp. 333-341. Apr. 2004.

Barabási, A. L. (2002). Statistical mechanics of complex networks. Reviews of Modern Physics, 74:51.

Barabási, A.-L. (2005). The origin of bursts and heavy tails in human dynamics. Nature, 435(7039):207-211. 10.1038/nature03459.

Brockmann, D., Hufnagel, L., and Geisel, T. (2006). The scaling laws of human travel. Nature, 439(7075):462-465. 10.1038/nature04292.

Campanharo, A. S. L. O., Sirer, M. I., Malmgren, R. D., Ramos, F. M., and Amaral, L. A. N. (2011). Duality between time series and networks. PLOS ONE, 6(8):e23378. 
Chechik, G., Oh, E., Rando, O., Weissman, J., Regev, A., and Koller, D. (2008). Activity motifs reveal principles of timing in transcriptional control of the yeast metabolic network. Nat Biotech, 26(11):1251-1259. 10.1038/nbt.1499.

Clarke, J. P., Durand, N., Feron, E., Singhose, W., and Vela, A. (2011). Determining the value of information for minimizing controller taskload: A graph-based approach.

DONNER, R. V., Donges, J. F., Marwan, N., and Zou, Y. (2010). Recurrencebased time series analysis by means of complex network methods. International Journal of Bifurcation and Chaos (IJBC), 21(4):1019-1046.

Erdös, P. and Réyi, A. (1959). On random graphs. Publicationes Mathematicae, 6:8.

Galy, E., Cariou, M., and Mlan, C. (2012). What is the relationship between mental workload factors and cognitive load types? International Journal of Psychophysiology, 83(3):269 - 275.

Gautreau, A., Barrat, A., and Barth?emy, M. (2009). Microdynamics in stationary complex networks. Proceedings of the National Academy of Sciences, 106(22):8847-8852.

Gonzalez, M. C., Hidalgo, C. A., and Barabási, A.-L. (2008). Understanding individual human mobility patterns. Nature, 453(7196):779-782. 10.1038/nature06958.

Gravio, G. D., Mancini, M., Patriarca, R., and Costantino, F. (2015). Overall safety performance of air traffic management system: Forecasting and monitoring. Safety Science, 72:351 - 362.

Guo, R., Zhang, Y., and Wang, Q. (2014). Comparison of emerging ground propulsion systems for electrified aircraft taxi operations. Transportation Research Part C: Emerging Technologies, 44:98 - 109.

Haraguchi, Y., Shimada, Y., Ikeguchi, T., and Aihara, K. (2009). Transformation from complex networks to time series using classical multidimensional scaling. In Alippi, C., Polycarpou, M., Panayiotou, C., and Ellinas, G., editors, Artificial Neural Networks ?ICANN 2009 , volume 5769 of Lecture Notes in Computer Science, pages 325-334. Springer Berlin / Heidelberg.

Hering, H. (2001). Technical analysis of atc controller to pilot voice communication with regard to automatic speech recognition system. Technical report, EUROCONTROL Experimental Center.

Hilburn, B. (2004). Cognitive complexity in air traffic control literature review. Technical Report EEC Note No.04/04, Eurocontrol.

Histon, J. M. and Hansman Jr, R. J. (2008). Mitigating complexity in air traffic control: the role of structure-based abstractions. Technical Report ICAT-2008-05, ICAT.

Holme, P. and Saramäki, J. (2011). Temporal Networks. ArXiv e-prints.

Kupfer, M., Callantine, T., Martin, L., and Mercer, J. (2011). Controller support tools for schedule-based terminal-area operations.

Lacasa, L., Luque, B., Ballesteros, F., Luque, J., and Nuno, J. C. (2008). From time series to complex networks: The visibility graph. Proceedings of the National Academy of Sciences, 105(13):4972-4975.

Lacher, J., Battise, V., Koteskey, R., Dao, A.-Q. V., Brandt, S. L., Ligda, S. V., and Wu, S.-C. (2011). Issues for near-term implementation of trajectory based operations.

Lancichinetti, A., Radicchi, F., Ramasco, J. J., and Fortunato, S. (2011). Finding statistically significant communities in networks. PLoS ONE, 6(4):e18961.

Laudeman, I. V., Shelden, S., Branstrom, R., and Brasil, C. L. (1998). Dynamic density: An air traffic management metric. Technical Report NASA/TM-1998-112226, National Aeronautics and Space Administration, Ames Research Center;.

Li, L. and Hansman, R. J. (2009). Experimental studies of cognitively based air traffic control complexity metrics for future operational concepts. Technical Report ICAT-2009-03, International Center for Air Transportation.

Liben-Nowell, D. and Kleinberg, J. (2008). Tracing information flow on a global scale using internet chain-letter data. Proceedings of the National Academy of Sciences, 105(12):4633-4638.

Loft, S., Sanderson, P., Neal, A., and Mooij, M. (2007). Modeling and predicting mental workload in en route air traffic control: Critical review and broader implications. Human Factors, 49(3):376-399.

Malmgren, R. D., Stouffer, D. B., Campanharo, A. S. L. O., and Amaral, L. A. N. (2009). On universality in human correspondence activity. Science, 325(5948):1696-1700.

Malmgren, R. D., Stouffer, D. B., Motter, A. E., and Amaral, L. A. N. (2008). A poissonian explanation for heavy tails in e-mail communication. Proceedings of the National Academy of Sciences, 105(47):18153-18158.

Manning, C., Mills, S. H., Fox, C. M., Pfleiderer, E. M., and Mogilka, H. J. (2002). Using air traffic control taskload measures and communication events to predict subjective workload. Technical Report DOT/FAA/AM-02/4, Federal Aviation Administration.

Marwan, N., Donges, J. F., Zou, Y., Donner, R. V., and Kurths, J. (2009). Complex network approach for recurrence analysis of time series. Physics Letters A, 373(46):4246-4254.

Newman, M. E. J. (2012). Communities, modules and large-scale structure in networks. Nat Phys, 8(1):25-31. 10.1038/nphys2162.

Oliveira, J. G. and Barabási, A.-L. (2005). Human dynamics: Darwin and einstein correspondence patterns. Nature, 437(7063):1251-1251. $10.1038 / 4371251 \mathrm{a}$.

Prasad, M. and Gaikwad, A. (2015). Human error probability estimation by coupling simulator data and deterministic analysis. Progress in Nuclear Energy, 81:22-29.

Rocha, L. E. C., Liljeros, F., and Holme, P. (2010). Information dynamics shape the sexual networks of internet-mediated prostitution. Proceedings of the National Academy of Sciences.

Schmidt, D. K. (1978). A queuing analysis of the air traffic controller's work load. IEEE TRANSACTIONS ON Systems Man and Cybernetics, SMC-8(6):492-498.

Song, C., Koren, T., Wang, P., and Barabási, A.-L. (2010). Modelling the scaling properties of human mobility. Nat Phys, 6(10):818-823. 10.1038/nphys 1760 .

Sridhar, B., Sheth, K. S., and Grabbe, S. (1998). Airspace complexity and its application in air traffic management. In 2nd USA/Europe Air Traffic Management RE्ED Seminar, pages 1-6. 2 ndUSA/Europe Air Traffic Management R\&D Seminar.

Vassilis, K. (2009). Temporal graphs. Physica A: Statistical Mechanics and its Applications, 388(6):1007-1023.

Wang, Y., Vormer, F., Hu, M., and Duong, V. (2013). Empirical analysis of air traffic controller dynamics. Transportation Research Part C: Emerging Technologies, 33:203 - 213. 
Wang, Y., Zhang, Q., Zhu, C., Hu, M., and Duong, V. (2016). Human activity under high pressure: A case study on fluctuation scaling of air traffic controllers communication behaviors. Physica A: Statistical Mechanics and its Applications, 441:151 - 157.

Xu, X., Zhang, J., and Small, M. (2008). Superfamily phenomena and motifs of networks induced from time series. Proceedings of the National Academy of Sciences, 105(50):19601-19605.

Yang, S. and Hu, M. (2010). Estimation of air traffic longitudinal conflict probability based on the reaction time of controllers. Safety Science, 48(7):926-930.

Zhang, J. and Small, M. (2006). Complex network from pseudoperiodic time series: Topology versus dynamics. Physical Review Letters, 96(23):238701. PRL.

Zhao, Q., Tian, Y., He, Q., Oliver, N., Jin, R., and Lee, W.-C. (2010). Communication motifs: a tool to characterize social communications. In Proceedings of the 19th ACM international conference on Information and knowledge management, CIKM '10, pages 1645-1648, New York, NY, USA. ACM.

Zhou, T., Kiet, H., Kim, J. B., Wang, B.-h., and Holme, P. (2008). Role of activity in human dynamics. EPL, 82(28002). 